\title{
La diversité des vignobles français face au changement climatique : simulations climatiques et prospective participative
}

\author{
Nathalie Ollat ${ }^{1^{*}}$, Sébastien Zito ${ }^{2}$, Yves Richard ${ }^{2}$, Patrick Aigrain ${ }^{3}$, Françoise Brugière ${ }^{3}$, \\ Eric Duchêne ${ }^{4}$, Iñaki Garcia De Cortazar-Atauri ${ }^{5}$, Jacques Gautier ${ }^{6}$, Eric Giraud-Héraud ${ }^{7}$, \\ Hervé Hannin ${ }^{8}$, Jean-Marc Touzard ${ }^{9}$, Benjamin Bois ${ }^{2}$ \\ ${ }^{1}$ EGFV, Université de Bordeaux, Bordeaux Sciences Agro, INRAE, ISVV, F-33882 Villenave d'Ornon, France \\ ${ }^{2}$ Biogéosciences, CNRS/uB, Université Bourgogne-Franche-Comté, Dijon, France \\ ${ }^{3}$ FranceAgriMer, Montreuil, France \\ ${ }^{4}$ SVQV, Université de Strasbourg, INRAE, Colmar, France \\ ${ }^{5}$ Agroclim, INRAE, Avignon, France \\ ${ }^{6}$ INAO, Montreuil, France \\ ${ }^{7}$ Univ. Bordeaux, INRAE, BSE, UMR CNRS 6060, USC INRAE 1441, ISVV, F-33140 Villenave d'Ornon, France \\ ${ }^{8}$ Université de Montpellier, MOISA, Institut Agro Montpellier/IHEV, Montpellier, France \\ ${ }^{9}$ Université de Montpellier, Innovation, INRAE, Montpellier, France
}

\begin{abstract}
Résumé - Etant donné les spécificités locales de la viti-viniculture française, en partie liées aux conditions climatiques, le changement climatique est un défi majeur que les acteurs de cette filière doivent relever régionalement. Pour explorer les stratégies d'adaptation à cette échelle, l'article combine deux approches : $i$ ) une description et simulation climatique régionalisée et ii) une prospective participative avec les acteurs de ces vignobles. L'étude climatique décrit les évolutions, entre le passé récent, la période actuelle et le milieu du XXI siècle, de cinq indicateurs climatiques (Indice de Huglin, nombre de jours où la température maximale est supérieure à $35^{\circ} \mathrm{C}$, cumuls des précipitations d'avril à septembre et d'avril à juillet, nombre de jours de précipitations supérieures à $1 \mathrm{~mm}$ entre avril à juillet), régionalisés à l'échelle $8 \times 8 \mathrm{~km}$ dans neuf régions viticoles, à partir de 22 modèles GCM issus de l'exercice CMIP5. Dans ce contexte, les réactions de 500 acteurs sont présentées face à quatre scénarios d'adaptation de la filière d'ici 2050 (conservateur, innovant, nomade, libéral). Leurs perceptions et positionnements stratégiques ainsi que leurs propositions d'action ont été recueillis lors de forums participatifs organisés dans sept régions viticoles englobant les neuf espaces climatiques. Les évolutions climatiques sont marquées par une augmentation des températures et du nombre de jours très chauds, plus intense dans le quart nord-est sur la période historique, et dans le quart sud-est, y compris le Beaujolais, d'ici 2046-2065. L'analyse du positionnement des acteurs révèle la volonté de conserver le modèle socio-économique actuel, avec des spécificités régionales liées en partie à l'intensité des évolutions climatiques. Dans les sept régions, les acteurs sont majoritairement en faveur de l'intégration encadrée d'innovations, tout en étant conscients des risques économiques et de perte de références. Les propositions d'actions présentent des orientations communes, notamment sur l'enjeu de développer la $R \& D$, et des spécificités associées aux évolutions climatiques, mais aussi à la structure du vignoble et à la gouvernance de sa filière.
\end{abstract}

Mots-clés : indicateurs climatiques, évolutions climatiques, recherche participative, viticulture, adaptation.

\begin{abstract}
The diversity of French vineyards facing climate change: climate simulations and participative foresight. Given the local specificities of French viti-viniculture, partly linked to climatic conditions, climate change is a major challenge that stakeholders in the wine industry must take up regionally. To explore adaptation strategies at this scale, the article combines two approaches: $i$ ) a regionalized climate description and simulation and $i$ ) a participatory foresight with the stakeholders of the regional wine industries. The climate study describes the changes, between the recent past, the current period and the middle of the $21^{\text {st }}$ century, of five climate indicators (Huglin index, number of days when Tmax is greater than $35^{\circ} \mathrm{C}$, total precipitation from April to September and from April to July, number of days of precipitation greater than $1 \mathrm{~mm}$ between April to July), downscaled at the $8 \times 8 \mathrm{~km}$ scale in 9 wine-growing regions, using $22 \mathrm{GCM}$ models from the CMIP5 exercise. In
\end{abstract}


parallel, the reactions of 500 players are presented in the face of four adaptation scenarios for the wine industry by 2050 (conservative, innovative, nomadic, liberal). Their perceptions and strategic positions as well as their proposals for action were gathered during participatory forums organized in seven wine regions encompassing the nine climatic areas. Climatic changes are marked by an increase in temperatures and the number of very hot days, more intense in the Northeast quarter over the historical period, and in the Southeast quarter, including Beaujolais, by 2046-2065. The analysis of the positioning of the players reveals the willingness to maintain the current socio-economic model, with regional specificities linked in part to the intensity of climate change. In the seven regions, the players are mostly in favor of the supervised integration of innovations, while being aware of the economic risks and the loss of references. The action proposals present common orientations, in particular on the issue of developing $\mathrm{R} \& \mathrm{D}$ and specificities associated with climate change, but also the structure of the vineyard and the governance of the wine industry.

Keywords: climatic indices, climatic evolution, participatory research, wine industry, adaptation.

* $\overline{\text { Auteure de correspondance }}:$ nathalie.ollat@inrae.fr

\section{Introduction}

En venant bouleverser les systèmes de production et de valorisation des vins établis depuis le début du $\mathrm{XX}^{\mathrm{e}}$ siècle, le changement climatique représente un défi majeur pour la filière vitivinicole française, très liée à la notion de «terroir » et à des conditions socio-environnementales qui ancrent régionalement la production et sont fortement valorisées économiquement (Ollat et al., 2020).

Par rapport à la période 1976-2005, les simulations climatiques font état d'un réchauffement qui varie peu entre les différents scénarios d'émission d'ici $2040\left(+1\right.$ à $\left.1,5^{\circ} \mathrm{C}\right)$, mais pourrait atteindre, dans le sud-est de la France, plus de $5^{\circ} \mathrm{C}$ à la fin du $\mathrm{XXI}^{\mathrm{e}}$ siècle pour le scénario le plus pessimiste, avec une hausse spectaculaire du nombre de jours de forte chaleur (Soubeyroux et al., 2021). L'évolution des précipitations est caractérisée par une forte incertitude, mais les tendances actuelles pourraient s'accentuer avec une augmentation de la durée des épisodes de sécheresse météorologiques, notamment dans le sud et l'ouest de la France (Soubeyroux et al., 2021).

Déjà perceptibles depuis les années 1980, les conséquences attendues sur la vigne et le vin préoccupent les acteurs de la filière. Avancées des vendanges jusqu'à 20 à 30 jours d'ici la fin du XXI ${ }^{\mathrm{e}}$ siècle (Fraga et al., 2016), accroissements possibles des risques de gel de printemps (Sgubin et al., 2018), augmentations de température pendant la période de maturation des raisins affectant leur potentiel œnologique (Madelin et al., 2010 ; van
Leeuwen et Destrac-Irvine, 2017) : le constat et les projections sont plutôt inquiétants pour les régions viticoles traditionnelles qui craignent pour leur capacité à conserver des marchés et assurer leur durabilité économique. Cependant, dans certains vignobles, ces nouvelles conditions climatiques pourraient s'avérer moins pénalisantes, comme en Alsace (Duchêne et Schneider, 2005). Par ailleurs, de nouvelles potentialités viticoles pourraient apparaitre dans des régions où la viticulture est marginale (Ollat et al., 2016), par exemple en Bretagne (Bonnardot et Quénol, 2020).

Mobilisée dès les années 2000, la recherche a très tôt informé les acteurs de la filière viti-vinicole sur les effets potentiels du changement climatique et les risques associés. Elle a initié des études, notamment à l'INRAE, mais aussi au CNRS, dans les Universités et Grandes Ecoles, pour quantifier les évolutions observées et produire des simulations des conséquences attendues (Seguin et Garcia de Cortazar-Atauri, 2005 ; Briche et al., 2009). A partir de 2012, sous l'impulsion de l'INRAE, ces efforts ont été fédérés au sein du projet «Laccave » (Ollat et Touzard, 2014) afin de fournir des données plus précises, d'étudier de manière concertée et systémique des leviers d'adaptation et de mettre à disposition des acteurs de la filière des outils pour concevoir des stratégies d'adaptation, notamment via un exercice de prospective associant Montpellier SupAgro, FranceAgriMer et l'Institut National de l'Origine et de la qualité (Aigrain et al., 2016a).

La prospective est une démarche relevant des sciences de l'anticipation, basée sur le principe que 
l'avenir peut être en partie construit sur la base de la volonté des acteurs et de leur organisation. Elle se démarque de la prévision obtenue par simulation, et vise à ouvrir le champ des possibles pour le futur. C'est un exercice pluridisciplinaire, adapté à différentes échelles et à différents secteurs socioéconomiques, qui permet d'alimenter la réflexion de manière collective. Appropriées pour traiter de questions liées au développement durable et au changement climatique, y compris en agriculture, plusieurs études prospectives ont concerné le secteur vitivinicole français en déclinant à différentes échelles (régionales ou catégories de vin) une étude fondatrice (Sebillotte et al., 2003). Mais jusqu'en 2014, aucune n'avait clairement traité des enjeux climatiques (Hannin et al., 2021).

Dans un contexte de changement climatique, quatre scénarios pour la filière vigne et vin française à l'horizon 2050 ont été élaborés, en combinant deux axes d'adaptation que sont le degré d'innovation technique et le déplacement géographique des vignobles : 1) un scénario « conservateur » où les changements introduits par les acteurs sont mineurs, 2) un « innovant » qui privilégie les innovations techniques, 3) un « nomade » où la priorité est donnée à la mobilité géographique pour retrouver des conditions climatiques plus favorables, et 4) un « libéral » dans lequel tous les leviers de 1'adaptation peuvent être mobilisés (Aigrain et al., 2016b, 2019 ; Touzard et $a l ., 2020)$. Ces scénarios ont ensuite été présentés à plus de 500 acteurs de la filière dans le cadre de forums participatifs organisés dans sept régions viticoles. Lors de chaque forum, les participants ont réfléchi aux conséquences de chaque scénario d'avenir, se sont prononcés sur les attitudes stratégiques à adopter et ont émis plus de 2600 propositions d'actions. Ces propositions ont été finalement utilisées par un groupe national représentant les principales organisations de la filière pour élaborer une stratégie nationale d'adaptation au changement climatique (Aigrain et al., 2019 ; Ollat et al., 2020 ; Hannin et al., 2021).

L'objectif de cet article est de montrer comment une étude décrivant des évolutions climatiques régionalisées peut être associée à une prospective participative réalisée avec les acteurs professionnels de vignobles régionaux, pour explorer et questionner leurs perceptions du changement climatique et leurs visions des adaptations nécessaires. En s'appuyant sur les réactions de ces acteurs recueillies lors de forums participatifs organisés dans sept régions viticoles, nous souhaitons mettre en évidence le caractère générique et les spécificités régionales de leurs perceptions et positionnements, et les confronter aux résultats d'études climatiques régionalisées. Après une présentation des méthodes utilisées pour l'étude climatique sur la base de données historiques et simulées pour le milieu $\mathrm{XXI}^{\mathrm{e}}$ siècle, et pour l'organisation des forums régionaux et la collecte des contributions des participants, les résultats sont présentés, puis confrontés et discutés au regard d'autres travaux abordant, généralement de manière partielle, les liens entre perception du contexte climatique des vignobles et options d'adaptation proposées par leurs acteurs.

\section{Données et méthodes}

\subsection{Données climatiques}

Les évolutions climatiques ont été étudiées pour les sept régions concernées par les forums participatifs. Mais pour mieux rendre compte des gradients nord-sud, les espaces climatiques ont été scindés en deux dans deux cas : la Bourgogne et le Beaujolais qui avaient fait l'objet d'un même forum participatif, tout comme les Côtes du Rhône septentrionales et méridionales. Ainsi, 9 espaces viticoles ont été étudiés en s'appuyant sur des données climatiques régionalisées à $8 \mathrm{~km}$ de résolution (figure $1 \mathrm{~A}$ ).

Pour la période 1980-2019, les données climatiques proviennent de la base de données SAFRAN (Système d'Analyse Fournissant des Renseignements Adaptés à la Nivologie) produite par le service météorologique national français (Le Moigne, 2002). Le système SAFRAN combine des observations issues de stations météorologiques ainsi que les sorties de modèles de prévisions météorologiques (modèle ARPÈGE) et fournit des données régionalisées à une résolution spatiale de 8 $\mathrm{km}$. Les données de températures minimales, maximales ainsi que les précipitations quotidiennes sont utilisées sur la période 1980-2019.

Pour la période 2046-2065, l'ensemble des 
projections climatiques utilisées correspond aux données des modèles dits de circulation générale (GCM) de l'exercice CMIP5 (Coupled Model Intercomparison Project : Taylor et al., 2012) mobilisées dans le cinquième rapport d'évaluation du GIEC. Au total, les données de 22 GCM, désagrégés et débiaisés statistiquement à partir de la méthode de «quantile mapping » (Gudmundsson et al., 2012) à l'échelle spatiale des données SAFRAN, ont été reprises (dataosu.obs-besancon.fr/ FR-18008901306731-2021-04-12-02 Climate-projectionover-France-8-km-resolution.html). Les détails concernant le processus de désagrégation et l'évaluation de la qualité des données climatiques qui en sont issues sont présentés dans la thèse de Zito (2021).
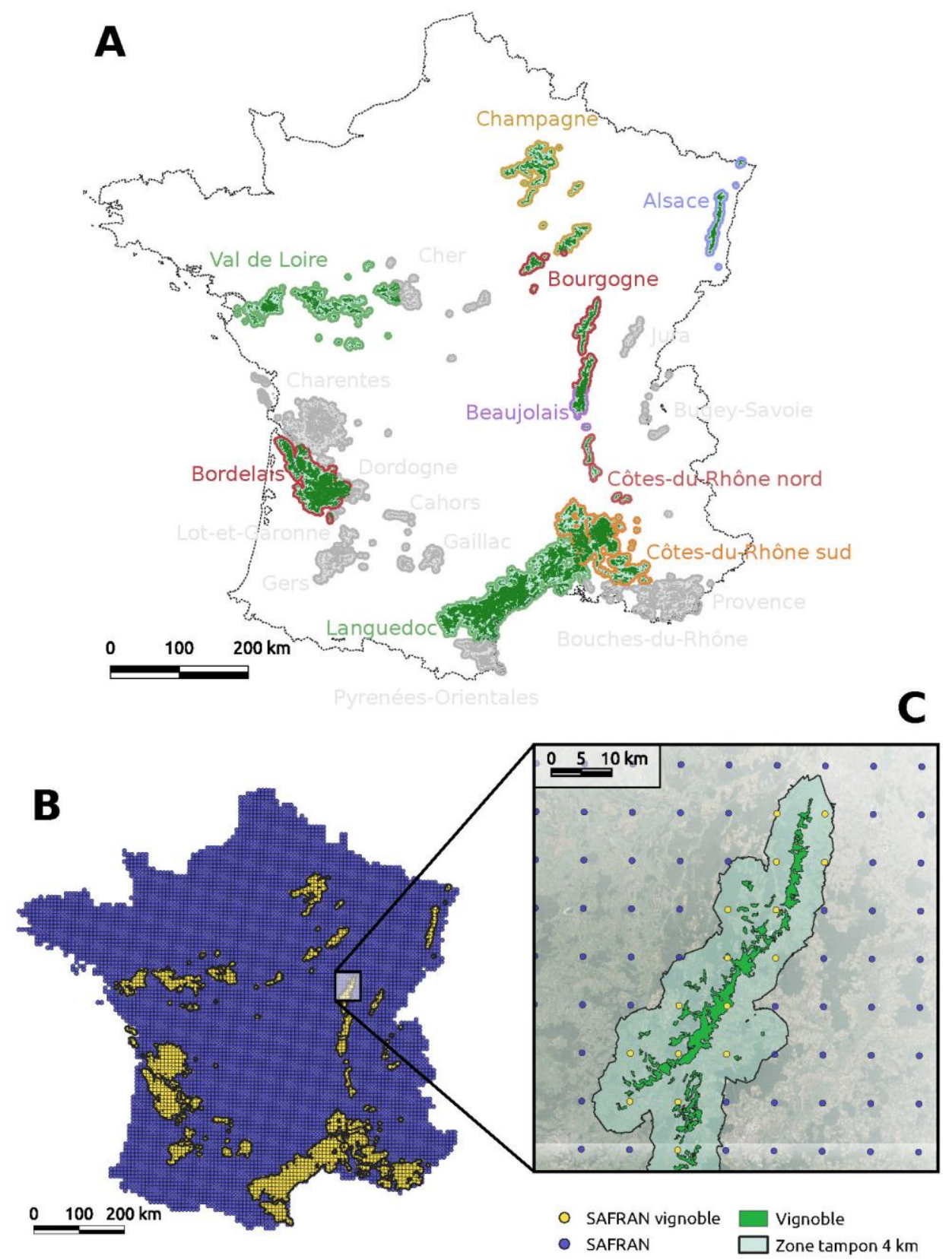

Figure 1. La carte A présente les régions viticoles françaises définies par Simonovici (2019), en délimitant en couleur les 9 régions retenues pour l'étude climatique. La carte B indique les points de grille SAFRAN correspondants, et la carte $\mathrm{C}$ présente un exemple de sélection des données climatiques SAFRAN (zone tampon de $4 \mathrm{~km}$ appliquée sur le vignoble). The map A displays the French wine regions defined by Simonovici (2019). The 9 coloured regions are those selected for the climatic study. Map B shows the corresponding SAFRAN grid points, and map $C$ shows an example of the selection of SAFRAN climatic data (4 km buffer zone applied to the vineyard). 
A partir de cette base de données climatiques régionalisées à $8 \mathrm{~km}$ (figure $1 \mathrm{~B}$ ), un souséchantillon des données correspondant aux régions vitivinicoles a été extrait, en s'appuyant sur la carte des bassins vitivinicoles français (Simonovici, 2019) digitalisée et géoréférencée, et en utilisant une identification des zones plantées en vignes fournie par la classification Corine Land Cover (CLC) de 2012. Afin de sélectionner les points de grille SAFRAN représentatifs des zones viticoles, une zone tampon de $4 \mathrm{~km}$ a été ajoutée aux limites des polygones (figure 1C).

Les périodes 1980-1999 et 2000-2019 ont été sélectionnées afin d'étudier l'évolution du climat entre le passé récent et la période actuelle. Des périodes de 20 ans, régulièrement rapportées dans la littérature scientifique (GIEC, 2021) ont été préférées à des durées de 30 ans (durée standard reconnue internationalement pour le calcul des normales climatiques) pour capturer l'évolution récente du climat en intégrant sa nature nonstationnaire, en raison, entre autres, du changement climatique contemporain. De plus, cela a permis de comparer le climat récent et les projections futures sur le même pas de temps. Concernant les projections futures, la période 2046-2065 (en comparaison à la période historique 1980-1999) a été choisie pour caractériser l'évolution potentielle des indicateurs pour le milieu du $\mathrm{XXI}^{\mathrm{e}}$ siècle selon la trajectoire RCP8.5. Cette période correspond à l'horizon de temps pour lequel l'exercice de prospective a été mené. Le scénario d'émission retenu correspond le mieux aux émissions de $\mathrm{CO}_{2}$ historiques à date, et semble en 2020 cohérent avec les politiques adoptées vis-à-vis des émissions de gaz à effet de serre à l'échelle planétaire (Schwalm et al., 2020).

\subsection{Indicateurs agroclimatiques}

Afin de décrire l'évolution des conditions climatiques sur les différentes périodes étudiées, plusieurs indicateurs agroclimatiques ont été retenus. Ils correspondent à des hypothèses climatiques qui ont été faites pour contextualiser l'approche prospective : augmentation générale des températures selon les scénarios du GIEC (peu différentes pour l'horizon de temps considéré), avec un différentiel entre le nord et le sud en matière de précipitations et de risques maladies. Ces indicateurs sont les suivants :

- l'indice héliothermique de Huglin (Huglin, 1978), correspondant au cumul de la moyenne des températures moyennes et maximales quotidiennes supérieures à $10^{\circ} \mathrm{C}$ (pendant la période du $1^{\mathrm{er}}$ avril au 30 septembre) multipliée par un coefficient de longueur du jour ( $\mathrm{k}$, variant selon la latitude). Par définition, il s'exprime en ${ }^{\circ} \mathrm{C}$.Jour $\left({ }^{\circ} \mathrm{C} . J\right)$. Cet indice, largement utilisé dans la littérature scientifique (Bois, 2020), décrit la précocité potentielle de la maturation du raisin ;

- le nombre de jours dont la température maximale dépasse $35^{\circ} \mathrm{C}$ (TASMAXs35). En effet, au-delà de cette température, la chaleur peut provoquer des dégâts observés au vignoble (Webb et al., 2009), plus particulièrement sur les raisins (Hulands et al., 2013 ; 2014) ;

- le cumul de précipitations sur la période avrilseptembre (GSPR). Il s'agit ici d'évaluer l'offre climatique en eau pour la vigne en saison végétative. Notons que cette approche simpliste ignore les conséquences de la fréquence et de l'intensité des précipitations, ainsi que la demande évaporative de l'atmosphère, qui permettraient de mieux décrire les potentialités de l'alimentation hydrique de la vigne. Le choix de ne pas intégrer la demande évaporative est délibéré. L'accès à la vitesse du vent en surface et au rayonnement global, variables essentielles pour estimer la demande évaporative (Bois et al., 2008) est limité à un petit nombre de modèles dans le cadre des simulations CMIP5. Aussi, l'estimation de la demande évaporative sur la seule base de la température pourrait conduire à la surévaluer (Bois, 2019) ;

- le cumul de précipitations (DSPR) sur la période d'avril à juillet inclus et le nombre de jours dont le cumul de précipitations est supérieur à $1 \mathrm{~mm}$ (DSPRs1) sur cette même période pendant laquelle la vigne est considérée comme sensible au mildiou et à l'oïdium, deux maladies d'importance majeure (Bois et al., 2017). Le cumul et le nombre de jours de pluie sur cette période ont été identifiés comme des variables guidant fortement la prévalence de l'oïdium en Bourgogne et en Champagne (Zito, 2021), le nombre de traitements contre le mildiou et l'oïdium en Bourgogne (Zito et al., 2018) et la précocité de l'épidémie de mildiou dans le nord de l'Italie 
(vignoble du Piave dans le Veneto : Salinari et al., 2007). Le nombre de jours de précipitations durant cette période renseigne également sur la possibilité de réaliser des travaux mécaniques et d'appliquer des traitements phytosanitaires, un temps sec étant nécessaire pour ces pratiques. Enfin, les traitements avec des produits phytosanitaires de contact (ne pénétrant pas dans les tissus de la plante et utilisés notamment en viticulture biologique de manière exclusive) sont rapidement lessivés par temps pluvieux (Pérez-Rodríguez et al., 2016).

La méthode appliquée permettant de caractériser l'évolution significative des indicateurs climatiques sélectionnés dans chacune des régions viticoles sur les différentes périodes (1980-1999 et 2000-2019 à l'aide des données SAFRAN et 2046-2065 avec les données CMIP5 désagrégées) suit les cinq étapes suivantes :

1. Calcul des indicateurs sur l'ensemble des points des grilles sélectionnés ;

2. Utilisation du test $t$ de Student (au risque $\alpha=5 \%$ ) afin d'étudier les différences significatives entre les périodes pour chacun des points de grille au sein des régions viticoles ;

3. Calcul de la moyenne des points de grille à l'échelle de chaque région viticole pour chacun des indicateurs ;

4. Dans le cas des données historiques (19801999 et 2000-2019), attribution d'une évolution significative de l'indicateur quand plus de $50 \%$ des points de grille de la région présente une différence significative (selon le test $t$ ) ;

5. Dans le cas des données pour la période 20462065, attribution d'une évolution significative de l'indicateur quand plus de $50 \%$ des modèles climatiques ont plus de $50 \%$ de points de grille de la région avec une différence significative (selon le test $t$ ).

Cette méthode permet ainsi de considérer à la fois la variabilité spatiale présente au sein des régions viticoles et la variabilité inter-modèles liée à la projection des différents indicateurs sur la période 2046-2065 pour le scénario d'émission RCP8.5. Ainsi, chaque modèle climatique est considéré et une évolution potentielle significative des indicateurs est prise en compte quand celle-ci est partagée pour une majorité des GCM utilisés.

\subsection{Forums participatifs dans sept régions viticoles}

\subsubsection{Organisation des forums régionaux}

Les récits correspondant aux quatre scénarios d'adaptation au changement climatique de la filière vitivinicole d'ici 2050 (conservateur, innovant, nomade, libéral) avaient été élaborés au niveau national entre 2014 et 2016 par le groupe de prospective en combinant 70 hypothèses d'ordre technique, économique, politique, interne et externe au système viti-vinicole (Aigrain et al., 2016c). Lors des forums participatifs, ces récits ont été présentés et discutés avec des acteurs des sept principales régions viticoles françaises. Chaque forum s'est déroulé sur une journée en Bordelais (Bordeaux 24/11/2016) avec des participants du grand Sud-Ouest (de Gaillac à Cognac), en Champagne (Epernay 23/03/2017), en Bourgogne et Beaujolais (Macon 28/03/2017), dans le Languedoc (Montpellier 22/11/2017) et les Côtes du Rhône-Provence (Avignon 23/11/2017), en Alsace (Colmar 06/12/2017) et Val de Loire (Saumur 07/03/2019). Chaque forum a été mis en place en collaboration avec les organisations professionnelles viticoles, en tenant compte de leurs spécificités régionales (Boyer et Touzard, 2016), soit au minimum l'interprofession régionale, associée selon les cas à un ou des syndicats d'appellation, un institut technique (IFV) ou une chambre d'agriculture. Les invitations ont été diffusées par ces organisations et les centres INRAE de chaque région en cherchant à rassembler une diversité d'acteurs de la filière, avec une limite maximale de 100 participants par forum. Pour chaque forum, les participants ont travaillé par groupes de 6 à 8 personnes établis par les organisateurs en recherchant une diversité de catégories professionnelles (vignerons, fournisseurs, négociants, services de conseil technique, recherche, administration). Des tablettes électroniques ont été mises à disposition de chaque groupe afin d'enregistrer et transmettre en temps réel les verbatims des participants, puis leurs votes, et pouvoir les synthétiser et restituer les retours de manière interactive à la fin des différentes séquences du forum. 


\subsubsection{Déroulement des forums}

Chaque forum d'une journée a été organisé selon les séquences suivantes :

- une présentation générale de la démarche prospective et des impacts régionaux du changement climatique tels que décrits dans cet article.

- un exposé de chaque scénario d'adaptation de la filière, suivi, pour chaque scénario, d'un temps d'échange de 20 minutes par groupe pour préciser ses conséquences en 2050 à l'échelle régionale. Les conséquences envisagées devaient concerner les domaines techniques (encépagement, pratiques...) et de marché (types de vin, commercialisation...), mais aussi les impacts pour les parties prenantes, les territoires et la gouvernance de la filière régionale. Chaque groupe a enregistré informatiquement sous forme de verbatims ces conséquences en les qualifiant comme positives, négatives ou neutres. Ces relevés textuels ont été transmis à l'équipe de prospective, chargée de réaliser dans la foulée leurs regroupements thématiques et leurs synthèses. En deux heures, les groupes ont ainsi exploré et spécifié les 4 scénarios d'adaptation pour leur région.

- une restitution des synthèses des verbatims pour chaque scénario, sous la forme de tableaux indiquant les thèmes récurrents et originaux par domaine d'enjeux et de conséquences. La restitution pour chaque scénario était suivie d'un temps d'échange avec l'ensemble des participants pour commenter et compléter les retours.

- une étape de vote pendant laquelle les participants ont été invités à sélectionner individuellement une attitude stratégique pour chaque scénario : proactivité positive ou négative (agir dès aujourd'hui pour favoriser ou défavoriser un scénario), réactivité anticipée (se préparer à agir), veille ou indifférent. Les votes étaient aussitôt affichés et commentés par l'équipe prospective.

- un temps de recueil de propositions d'actions émises par chaque participant pour la mise en œuvre de l'attitude stratégique choisie sur chaque scénario. Ces propositions, libres dans leurs formulations (généralement une phrase) ont été recueillies sur papier pour des analyses ultérieures.
- une conclusion du forum par l'intervention d'un grand témoin, puis d'un débat général entre les participants.

\subsubsection{Analyses des données}

Les informations collectées lors des sept forums régionaux ont été saisies et agrégées pour construire deux bases de données. La première a été constituée à partir des verbatims «enjeux et conséquences », caractérisés par leur appréciation (positive, négative, neutre), le numéro du groupe de travail qui les a énoncés et la région viticole concernée. $\mathrm{La}$ deuxième base, plus importante, a été structurée à partir de l'ensemble des propositions d'actions recueillies en fin de journée, décrites par un verbatim (ex. " développer l'irrigation dans le vignoble languedocien »), un des quatre scénarios (ex. nomade), l'attitude stratégique vis-à-vis de ce scénario (ex. veille), un numéro de participant (chaque participant étant libre de formuler plusieurs propositions), ses caractéristiques professionnelles et sa région.

Des analyses qualitatives ont été ensuite réalisées par le groupe de prospective pour établir une catégorisation des verbatims de chaque base (ex. domaines et sous-domaines d'actions) ou sélectionner des verbatims jugés collectivement illustratifs de chaque catégorie. Des analyses et tests statistiques ont ensuite été effectués pour caractériser les réactions des participants (répartition par catégorie, croisements entre attitudes stratégiques et scénarios...) et repérer les variables pouvant discriminer les régions, dont les évolutions climatiques ont été précisées en parallèle (cf. §1.1 et 1.2).

\section{Résultats}

\subsection{Analyse du contexte climatique}

\subsubsection{Période climatique historique}

Une élévation significative de l'indice héliothermique de Huglin est mise en évidence entre les périodes 1980-1999 et 2000-2019, dans toutes les régions viticoles présentées (tableau 1). Les régions de l'est/nord-est de la France sont les plus marquées par le réchauffement récent. Le Beaujolais affiche la hausse la plus importante $\left(236^{\circ} \mathrm{C} . J\right)$, suivie par l'Alsace et la Champagne 
(passant respectivement de $1459^{\circ} \mathrm{C} . \mathrm{J}$ à $1669^{\circ} \mathrm{C} . J$ et de 1367 à $\left.1577^{\circ} \mathrm{C} . J\right)$. Ce changement de classe a déjà été rapporté pour la Champagne sur la base de l'étude de données issues de la station climatique de Reims en Champagne entre 1978 et 2006 (Beltrando et Briche, 2010). En quarante ans, ces deux régions sont passées de la classe "très frais" à "frais" selon la classification des climats viticoles mondiaux proposée par Tonietto et Carbonneau (2004). La hausse de $175^{\circ} \mathrm{C} . J$ de l'indice de Huglin dans la partie septentrionale des Côtes du Rhône conduit à classer cette région pour la première partie du $\mathrm{XXI}^{\mathrm{e}}$ siècle comme région à climat "tempéré" ( $1897^{\circ}$ C.J en moyenne sur la période 2000-2019). La partie sud des Côtes de Rhône passe quant à elle dans la classe de climat "tempéré-chaud" $\left(2243^{\circ} \mathrm{C} . \mathrm{J}\right.$ en moyenne sur la période 2000-2019). C'est le cas également du Languedoc $\left(+183^{\circ} \mathrm{C}\right.$.J pour atteindre $2276^{\circ} \mathrm{C}$.J au début du $\mathrm{XXI}^{\mathrm{e}}$ siècle). Avec des hausses respectives de $176^{\circ} \mathrm{C} . J, 127^{\circ} \mathrm{C} . \mathrm{J}$ et $133^{\circ} \mathrm{C} . J$, la Bourgogne, le Bordelais et le Val de Loire se positionnent en début de $\mathrm{XXI}^{\mathrm{e}}$ siècle dans la partie supérieure de leur classe climatique actuelle, respectivement "frais", "tempéré" et "frais".

Tableau 1. Variation moyenne des indicateurs climatiques par rapport à la valeur moyenne de référence 1980-1999 (entre parenthèses) entre les périodes 2000-2019 et 1980-1999. Acronymes utilisés : Huglin $\left[{ }^{\circ} \mathrm{C} . \mathrm{J}\right]=$ indice héliothermique de Huglin ; TASMAXs35 = nombre de jours dont la température maximale dépasse $35^{\circ} \mathrm{C}$; GSPR [mm] = cumul de précipitations d'avril à septembre $; \mathrm{DSPR}[\mathrm{mm}]=$ cumul de précipitations d'avril à juillet $;$ DSPRs1 = nombre de jours d'avril à juillet dont le cumul de précipitations est supérieur ou égal à $1 \mathrm{~mm}$. Valeurs en gras : plus de $50 \%$ des points de grille de la région viticole présentent une évolution moyenne significative $(95 \%)$ entre les 2 périodes. Les valeurs correspondent à la moyenne des différences sur l'ensemble des points de grille de la région viticole. Average difference of climate indicators between the periods 2000-2019 and 1980-1999 (average absolute value in bracket for 1980-1999. Acronyms used: Huglin ${ }^{\circ}$ C.days] = Huglin heliothermic index; TASMAXs35 = number of days with a maximum temperature above $35^{\circ} \mathrm{C}$; GSPR $[\mathrm{mm}]=$ cumulative precipitation from April to September; $D S P R$ [mm] = cumulative precipitation from April to July; DSPRs $1=$ number of days from April to July for which the cumulative rainfall is greater than or equal to $1 \mathrm{~mm}$. Values in bold: More than $50 \%$ of the grid points in the wine-growing region show a significant average change (95\%) between the 2 periods. The values correspond to the average of the differences over all the grid points in the wine-growing region.

\begin{tabular}{|c|c|c|c|c|c|}
\hline Régions & Huglin & TASMAXs35 & GSPR & DSPR & DSPRs1 \\
\hline Alsace & $+210(1459)$ & $+0,5(0,1)$ & $-2,3(411,2)$ & $-8(286)$ & $-3,2(49,1)$ \\
\hline Beaujolais & +236 (1554) & $+0,8(0,3)$ & $-36,9(470,4)$ & $-20(317)$ & $-4,1(44,2)$ \\
\hline Bordelais & $+127(1880)$ & $+0,5(1)$ & $-41,6(386)$ & $-14(257)$ & $-3,4(41,2)$ \\
\hline Bourgogne & $+176(1550)$ & $+0,8(0,4)$ & $-25(448,9)$ & $-11(305)$ & $-2,1(44,6)$ \\
\hline Champagne & $+210(1367)$ & $+0,5(0,3)$ & $-7,8(375,4)$ & $-7(256)$ & $-2,9(44,2)$ \\
\hline Côtes-du-Rhône nord & +175 (1722) & $+1,2(0,4)$ & $-49(490)$ & $-27(318)$ & $-2,8(37,5)$ \\
\hline Côtes-du-Rhône sud & +185 (2058) & $+1,8(1,3)$ & $-38(391)$ & $-23(240)$ & $-2,4(26,5)$ \\
\hline Languedoc & $+183(2093)$ & $+1,8(0,9)$ & $-13,5(319,1)$ & $-5(199)$ & $-0,6(24,9)$ \\
\hline Val de Loire & +133 (1609) & $+0,6(0,4)$ & $-9,1(310,6)$ & +3 (208) & $-2,2(37,2)$ \\
\hline
\end{tabular}

Dans toutes les régions, le nombre de jours de fortes chaleurs $\left(\operatorname{Tmax}>35^{\circ} \mathrm{C}\right)$ marque une très légère hausse, mais sans aucune significativité statistique, en raison de la forte variabilité interannuelle (figure supplémentaire 1). Enfin, à l'exception de la baisse du nombre de jours de précipitations d'avril à juillet (DSPRs1) dans le Beaujolais, aucun autre indicateur agroclimatique ne présente de différence significative entre la fin du $\mathrm{XX}^{\mathrm{e}}$ siècle et le début du XXI ${ }^{\mathrm{e}}$ siècle. L'absence de signal pluviométrique significatif entre avril et septembre comme entre avril et juillet est en accord avec de nombreux travaux scientifiques listés dans le sixième rapport d'évaluation du GIEC (2021). Aucune évolution tendancielle des cumuls saisonniers de précipitations en Europe occidentale n'est en effet observée entre la fin du $\mathrm{XIX}^{\mathrm{e}}$ siècle et les deux premières décennies du $\mathrm{XXI}^{\mathrm{e}}$ siècle (Vicente-Serrano et al., 2021). En outre, de récents travaux rapportent l'absence de lien entre le forçage anthropique et l'évolution des précipitations en Europe méditerranéenne occidentale depuis la fin du $\mathrm{XIX}^{\mathrm{e}}$ siècle (de la Croatie au Portugal), dont les oscillations rapportées seraient davantage le fruit de la variabilité décennale ou interannuelle (Peña-Angulo et al., 2020). 


\subsubsection{Evolution climatique simulée pour la période 2046-2065}

Les projections à horizon 2050, pour le scénario RCP8.5 et en moyenne pour 22 modèles, révèlent une élévation nette et significative des deux indicateurs agroclimatiques fondés sur la température (tableau 2) et ce, pour chacun des 22 GCM étudiés (figures 2 et 3). L'indice de Huglin progresserait sensiblement : avec une augmentation de $662^{\circ} \mathrm{C} . J$ (Val de Loire) à $771^{\circ} \mathrm{C} . J$ (Côtes du Rhône méridionales), les régions viticoles françaises étudiées deviendraient des régions aux climats "tempéré chaud" (Alsace, Beaujolais, Bourgogne, Champagne et Val de Loire) à "chaud" (Bordelais, Languedoc, Côtes-du-Rhône septentrionales et méridionales), selon la classification proposée par Tonietto et Carbonneau (2004). Les valeurs moyennes de l'indice de Huglin dans le Bordelais, le Languedoc ou les Côtes du Rhône excèderaient les valeurs moyennes rapportées dans la Mancha en Espagne ou encore à Porto pour la période 19712000 (Jones et al., 2009).

Tableau 2. Projection des indicateurs climatiques pour le milieu du XXIe siècle (moyenne des différences des 22 GCM) entre les périodes 2046-2065 et 1980-1999 (valeur absolue de référence entre parenthèses) avec la trajectoire RCP8.5. Acronymes utilisés : Huglin = indice héliothermique de Huglin ; TASMAXs35 = nombre de jours dont la température maximale dépasse $35^{\circ} \mathrm{C}$; GSPR [mm] = cumul de précipitations d'avril à septembre ; DSPR [mm] = cumul de précipitations d'avril à juillet ; DSPRs1 = nombre de jours d'avril à juillet dont le cumul de précipitations est supérieur ou égal à $1 \mathrm{~mm}$. Valeurs en noir : plus de $50 \%$ des points de grille de la région viticole présentent une évolution moyenne significative $(95 \%)$ entre les 2 périodes pour plus de $50 \%$ des modèles utilisés. Projection of climate indicators for the middle of the 21st century (average of the differences of the 22 GCMs) between the periods 20462065 and 1980-1999 (reference absolute value in brackets) with the RCP8.5 trajectory. Acronyms used: Huglin = Huglin heliothermic index; TASMAXs35 = number of days with a maximum temperature above $35^{\circ} \mathrm{C} ; \mathrm{GSPR}[\mathrm{mm}]=$ cumulative precipitation from April to September; DSPR $[\mathrm{mm}]=$ cumulative precipitation from April to July; DSPRs1 = number of days from April to July for which the cumulative rainfall is greater than or equal to $1 \mathrm{~mm}$. Values in black: More than $50 \%$ of the grid points of the wine-growing region show a significant average change (95\%) between the 2 periods for more than $50 \%$ of the models used.

\begin{tabular}{|c|c|c|c|c|c|}
\hline \multicolumn{6}{|c|}{$1980-1999$ vs $2046-2065$} \\
\hline Région & Huglin & TASMAXs35 & GSPR & DSPR & DSPRs1 \\
\hline Alsace & $+679(1459)$ & $+5,8(0,1)$ & $+13(411)$ & $+15(286)$ & $-3(49,1)$ \\
\hline Beaujolais & $+752(1554)$ & $+11,3(0,3)$ & $-20(470)$ & $-10(317)$ & $-4,7(44,2)$ \\
\hline Bordelais & $+663(1880)$ & $+13(1)$ & $-41(386)$ & $-26(257)$ & $-6,4(41,2)$ \\
\hline Bourgogne & $+708(1550)$ & $+9,7(0,4)$ & $-8(449)$ & +1 (305) & $-4,1(44,6)$ \\
\hline Champagne & $+669(1367)$ & $+7,2(0,3)$ & $-11(375)$ & $-3(256)$ & $-4,2(44,2)$ \\
\hline Côtes-du-Rhône nord & $+737(1722)$ & $+13,9(0,4)$ & $-8(490)$ & $-7(318)$ & $-3,6(37,5)$ \\
\hline Côtes-du-Rhône sud & $+771(2058)$ & $+22,6(1,3)$ & $-18(391)$ & $-25(240)$ & $-3,7(26,5)$ \\
\hline Languedoc & $+741(2093)$ & $+21,3(0,9)$ & $-17(319)$ & $-25(199)$ & $-3,9(24,9)$ \\
\hline Val de Loire & $+662(1609)$ & $+8,4(0,4)$ & $-31(311)$ & $-18(208)$ & $-5,7(37,2)$ \\
\hline
\end{tabular}

Le nombre de jours de forte chaleur (TASMAXs35, température maximale excédant $35^{\circ} \mathrm{C}$ ) augmenterait également de façon significative pour l'ensemble des régions viticoles étudiées. Presque inexistants à la fin du $\mathrm{XX}^{\mathrm{e}}$ siècle en Alsace, on en compterait 5,8 en moyenne sur la période 2046-2065. Au début du $\mathrm{XXI}^{\mathrm{e}}$ siècle (période 2000-2019), aucune région viticole en France ne présente un nombre de jours de forte chaleur aussi élevé. En Champagne, le nombre de jours de forte chaleur s'élèverait de 7,2 jours par an en moyenne en référence à la période 1980-1999. En s'appuyant sur le seul modèle Arpège-Climat avec une maille de $50 \mathrm{~km}$ couvrant une large partie $\mathrm{du}$ vignoble champenois, Beltrando et Briche (2010) rapportent des simulations du nombre de jours de forte chaleur de 0 à 11 durant la période 2046-2065 de juin à août, selon les années et les anciens scenarios d'émission considérés (SRES B1, $\mathrm{A} 1 \mathrm{~B}, \mathrm{~B} 2$ de l'exercice CMIP3), correspondant à un nombre moyen de l'ordre de 1 à 3 jours par an (Briche, 2011). 

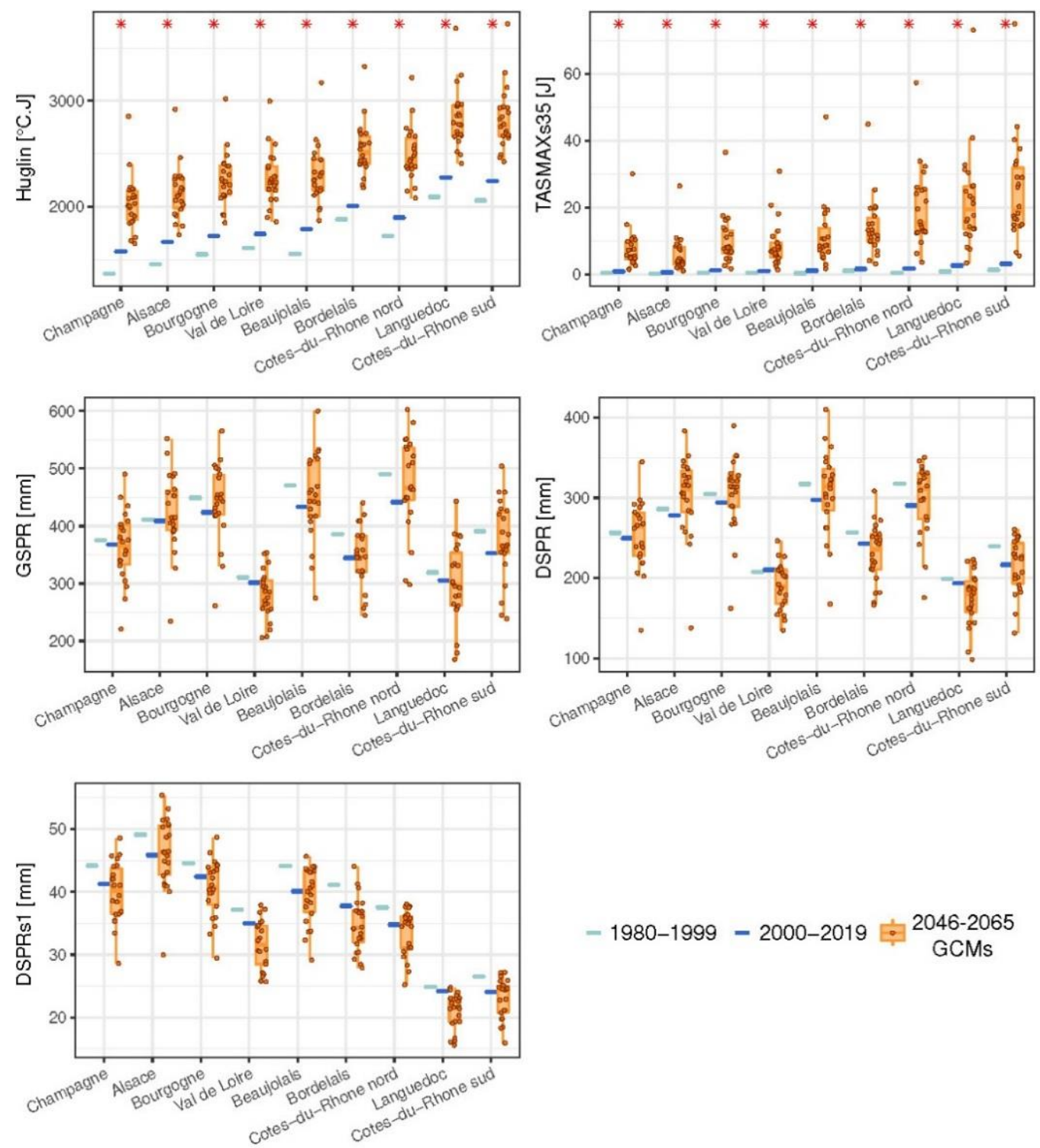

Figure 2. Dispersion statistique des valeurs d'indicateurs agroclimatiques moyens simulées par les 22 GCM sur la période 2046-2065 pour chacune des régions viticoles. Les traits bleus correspondent aux moyennes spatiotemporelles (points de grille et années) de chaque indicateur pour chaque région, calculé à partir des données historiques pour les périodes 1980-1990 (bleu clair) et 2000-2019 (bleu foncé). Chaque point rouge représente la moyenne spatio-temporelle (points de grille et années) de chaque indicateur pour chaque région, calculé pour chaque GCM sur la période 2046-2065. La dispersion entre les modèles est schématisée au moyen d'un box-plot. En ordonnées : Huglin $\left[{ }^{\circ} \mathrm{C} . \mathrm{J}\right]=$ indice héliothermique de Huglin ; TASMAXs35 = nombre de jours dont la température maximale dépasse $35^{\circ} \mathrm{C} ;$ GSPR $[\mathrm{mm}]=$ cumul de précipitation d'avril à septembre ; DSPR $[\mathrm{mm}]=$ cumul de précipitations d'avril à juillet ; DSPRs1 = nombre de jours d'avril à juillet dont le cumul de précipitations est supérieur ou égal à $1 \mathrm{~mm}$. Les astérisques rouges indiquent une évolution significative des indicateurs sur la période 2046-2065 en comparaison avec la période 1980-1999 pour plus de $50 \%$ des points de grilles sélectionnés au sein des régions viticoles. Statistical dispersion of the average agro-climatic index values simulated by the 22 GCMs over the period 2046-2065 for each of the wine-growing regions. The blue lines correspond to the spatio-temporal averages (grid points and years) of each index for each region calculated from historical data for the periods 1980-1990 (light blue) and 2000-2019 (dark blue). Each red dot corresponds to the spatiotemporal average (grid points and years) of each index for each region calculated for each GCM over the period 2046-2065. The dispersion between the models is shown schematically by means of a box-plot. On the $y$-axis: Huglin] = heliothermic index of Huglin; TASMAXs35 = number of days with a maximum temperature above $35^{\circ} \mathrm{C}$; GSPR $[\mathrm{mm}]=$ cumulative precipitation from April to September; $D S P R$ [mm] = cumulative precipitation from April to July; DSPRs1 = number of days from April to July for which the cumulative rainfall is greater than or equal to $1 \mathrm{~mm}$. The red asterisks indicate a significant change in the indicators over the period 2046-2065 compared to the period 1980-1999 for more than 50\% of the selected grid points within the wine-growing regions. 
Les Côtes du Rhône méridionales compteraient en moyenne 23 jours avec une température maximale supérieure à $35^{\circ} \mathrm{C}$ en 2050 . Les deux régions les plus chaudes (Languedoc et Côtes du Rhône sud) présenteraient un nombre de jours annuels de forte chaleur supérieur à 14, seuil employé pour définir les conditions limites de production de vins dits "prémium" dans l'Ouest des Etats-Unis (White et al., 2006). Ces conditions thermiques ne sont toutefois pas inédites en vitiviniculture. On retrouve actuellement dans de nombreuses régions vitivinicoles australiennes un nombre équivalent de jours de forte chaleur. Par exemple, la région viticole d'Adelaïde Hills (État de 1'Australie du Sud) affiche environ une dizaine de jours dont la température excède $35^{\circ} \mathrm{C}$ (Remenyi et al., 2019). Dans la vallée de Barossa (même État), ce seuil est dépassé environ vingt jours par an.

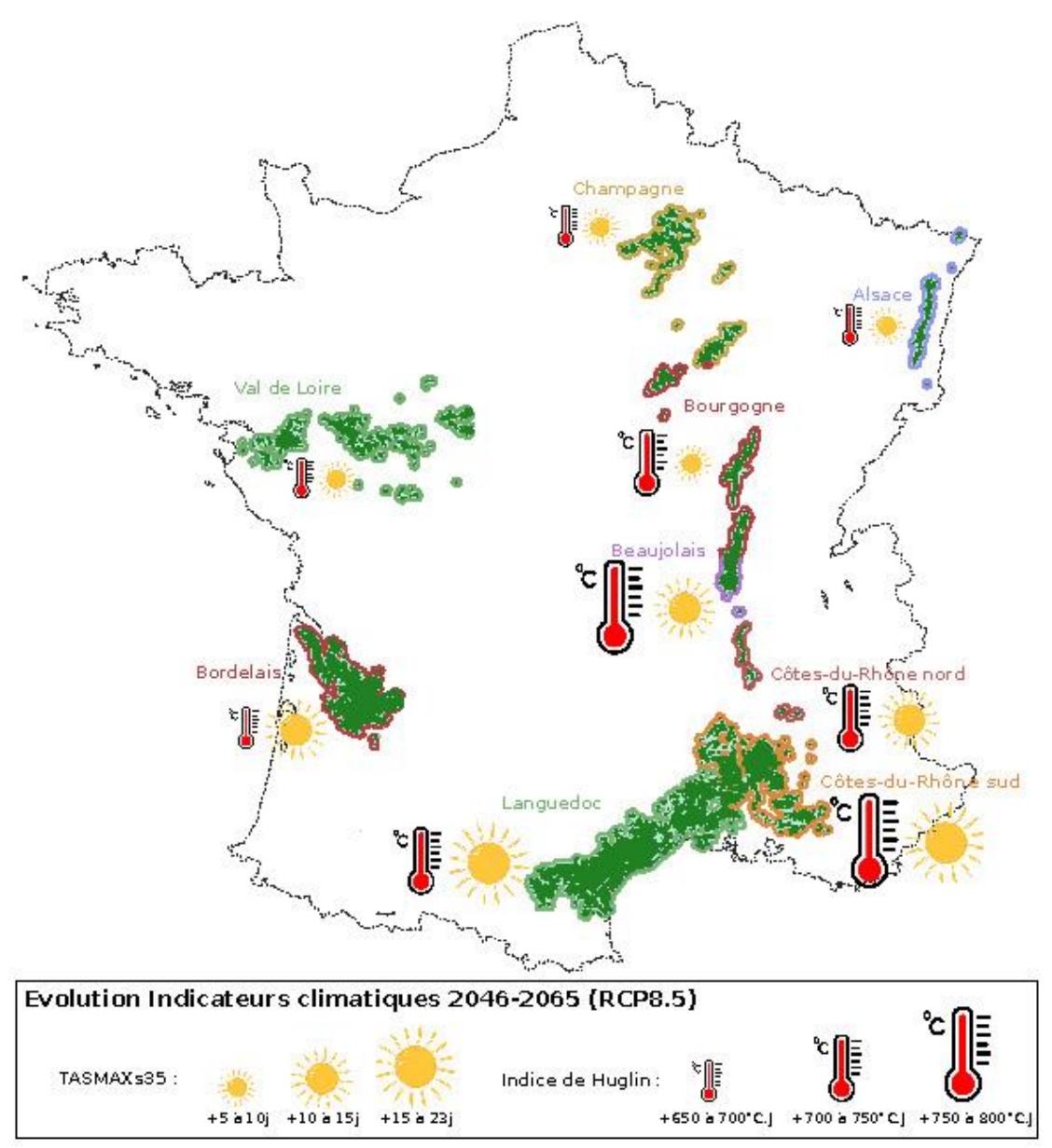

Figure 3. Iconographie illustrant les écarts entre la période 2046-2065 et la période 1980-1999 du nombre de jours de forte chaleur (TASMAXs35) et de l'indice héliothermique de Huglin $\left({ }^{\circ} \mathrm{C} . \mathrm{J}\right)$. Les icônes quantifient par leur taille les écarts significatifs entre les moyennes spatio-temporelle (tous les pixels SAFRAN de chaque région vitivinicole) des indices obtenus à partir des données climatiques désagrégées de $22 \mathrm{GCM}$ utilisés pour simuler le climat dans le cadre de l'exercice CMIP5 avec le scénario RCP8.5. Illustration of the range between the reference period 1980-1999 and the future period 2046-2065, for the number of very hot days (TASMAXs35) and the Huglin index ( $\left.{ }^{\circ} \mathrm{C} . J\right)$. The size of the small pictograms is proportional to the range between spatio-temporal means (for all the pixels of the SAFRAN grid for each wine region) of indicators calculated from the downscaled climatic data for 22 GCM used to simulate the climate (exercise CMIP5, scenario RCP8.5).

Les données de la présente étude simulent en moyenne une baisse des cumuls (GSPR d'avril à septembre et DSPR d'avril à juillet) et du nombre de jours de précipitations (DSPRs1 d'avril à juillet), et ce de façon plus marquée dans les vignobles occidentaux (Val de Loire et Bordelais). Cependant, ces tendances ne sont pas significatives pour tous ces indicateurs car des différences marquées entre les modèles de climat sont mises en évidence (figure 2). En effet, certains projettent des baisses de 
précipitations alors que d'autres simulent une augmentation des cumuls de pluie. La dispersion entre les sorties de GCM désagrégées ne permet donc pas de révéler une évolution statistiquement significative des indices agroclimatiques concernant les précipitations. L'absence de tendance significative pour les deux régions viticoles proches de la Mer Méditerranée (Languedoc et Côtes du Rhône méridionales) ne confirme pas les résultats publiés issus d'autres simulations qui rapportent une baisse des précipitations cumulées pour le pourtour méditerranéen, de manière concomitante avec l'élévation des températures au $\mathrm{XXI}^{\mathrm{e}}$ siècle (Lionello et Scarascia, 2018 ; Chan et al., 2020). En particulier, ces simulations s'accordent globalement sur une baisse des cumuls durant la saison estivale en Méditerranée et une hausse des précipitations en hiver pour le nord de l'Europe (GIEC, 2021). Une analyse de nos données, calée sur un horizon plus lointain avec le scénario RCP8.5 (données non présentées), confirme toutefois une réduction plus marquée du nombre de jours de pluie, en accord avec la littérature scientifique.
Dans le contexte des évolutions climatiques tendancielles majeures depuis les années 1980, décrites ci-dessus, nous avons interrogé des acteurs de la filière vitivinicole quant à leur vision des futurs possibles pour la production de vins dans leur région et leurs préférences en matière d'adaptation, sur la base de quatre scénarios prospectifs. Ils ont ainsi pu exprimer leurs perceptions des enjeux et conséquences de chaque scénario, puis leurs positionnements stratégiques et leurs propositions d'action au regard de ces scenarios.

\subsection{Perception climatique et réaction des acteurs face aux scénarios d'adaptation}

\subsubsection{Caractéristiques des participants aux forums}

Plus de 500 personnes ont participé aux sept forums régionaux, mais seulement 424 ont réellement contribué à l'ensemble des étapes de la journée (réactions, vote, propositions d'action), constituant la population analysée dans notre étude (tableau 3).

Tableau 3. Nombre et catégories professionnelles des participants aux forums ayant été actifs sur l'ensemble de la journée de travail dans chaque région. Number and professional category of actors who participated to the forums over the entire working day in each region.

\begin{tabular}{|c|c|c|c|c|c|c|c|c|c|}
\hline & 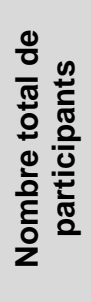 & 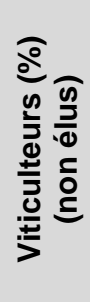 & 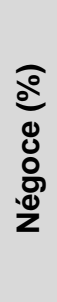 & $\begin{array}{l}\frac{0}{0} \\
y \\
\frac{d}{\frac{0}{0}} \\
\frac{5}{0} \\
\frac{0}{0} \\
0\end{array}$ & 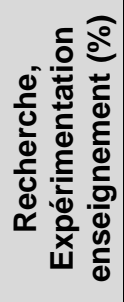 & 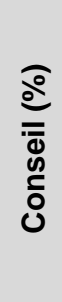 & 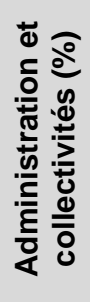 & 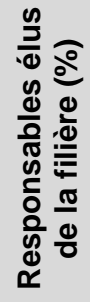 & $\frac{\precsim}{\stackrel{0}{\circ}}$ \\
\hline Alsace & 41 & 38 & 5 & 0 & 25 & 10 & 7 & 5 & 10 \\
\hline Bordelais & 60 & 20 & 7 & 2 & 31 & 9 & 5 & 19 & 7 \\
\hline Bourgogne-Beaujolais & 40 & 20 & 5 & 0 & 28 & 13 & 15 & 5 & 13 \\
\hline Champagne & 51 & 40 & 8 & 2 & 12 & 18 & 8 & 6 & 6 \\
\hline Côtes du Rhône & 78 & 35 & 6 & 0 & 12 & 19 & 11 & 3 & 14 \\
\hline Languedoc & 72 & 40 & 3 & 0 & 21 & 7 & 10 & 14 & 4 \\
\hline Val de Loire & 82 & 21 & 15 & 3 & 15 & 13 & 25 & 6 & 1 \\
\hline Nombre total & 424 & 32 & 7 & 1 & 18 & 13 & 13 & 8 & 8 \\
\hline
\end{tabular}

Le nombre de ces participants actifs a varié d'une région à l'autre, entre quarante (Alsace, Bourgogne) et quatre-vingt-deux (Val de Loire). Les catégories professionnelles présentes sont également variables, en particulier pour la part de viticulteurs n'ayant pas de mandat électif (entre $20 \%$ en Bourgogne-Beaujolais et $40 \%$ en Champagne) ou pour celles des acteurs liés à la $R \& D$ et l'enseignement (entre 12 \% en Côtes du Rhône et $30 \%$ pour le Bordelais). Sur l'ensemble des forums, 
un tiers des participants a déclaré intervenir au niveau de la production, un tiers pour des activités de recherche, conseil ou expérimentation et $20 \%$ pour des fonctions d'administration ou de gestion de la filière (interprofession par exemple). Le poids effectif des producteurs est de fait plus élevé (près de $40 \%$ ) si l'on inclut une partie des responsables d'organisations viticoles, souvent eux-mêmes viticulteurs. Le négoce et les pépiniéristes ont peu participé. Au final, les participants actifs par forum régional représentent une population diversifiée (avec seulement une sous-représentation du négoce et de fournisseurs), pertinente pour une analyse à une échelle nationale et pour des comparaisons interrégionales significatives sur certaines variables. Le biais d'engagement au regard de l'enjeu climatique n'est cependant pas à négliger. Les participants, inscrits volontairement, présentent en effet une motivation et des connaissances a priori plus affirmées sur ce thème que l'ensemble des acteurs de la filière. Une enquête menée en 2015 et 2016 auprès des viticulteurs du Bordelais, de Champagne et du Languedoc, montre en effet que si les préoccupations sont largement partagées sur le changement climatique, l'engagement d'actions d'adaptation des viticulteurs ne dépend pas des régions, mais de la formation et des liens de ces viticulteurs avec leurs organisations régionales (Boyer et Touzard, 2021).

\subsubsection{Perception du changement climatique et des conséquences des scénarios d'adaptation}

L'étude des verbatims recueillis permet de dégager les préoccupations climatiques et environnementales majeures des participants dans chaque région (citées le plus grand nombre de fois). Globalement, il ressort que l'ensemble des participants considère, quelles que soient les régions, que le changement climatique est associé à une augmentation des températures qui se traduit par une précocité accrue des vendanges, une augmentation de la teneur en alcool et une baisse d'acidité des raisins. Les participants citent également dans tous les forums la question de la raréfaction des ressources en eau.

Pourtant, des spécificités régionales ressortent quant à l'évaluation des risques climatiques. Les problèmes d'aléas climatiques sont plus souvent cités dans le Bordelais, en Val de Loire et en
Champagne. En Alsace et en Champagne, l'effet de l'élévation des températures apparait comme prépondérant. Dans ces deux régions, les risques d'accroissement des problèmes sanitaires (effet couplé d'augmentation des températures et des pluies) sont aussi beaucoup plus cités que dans les autres régions. La problématique de l'eau parait primordiale dans les Côtes du Rhône et en Languedoc où une très grande majorité de verbatims est relative à cette question. En Bourgogne et en Beaujolais, il ressort une vision transitoire entre le nord et le sud, avec des préoccupations voisines de celles de l'Alsace et de la Champagne, mais aussi avec des risques liés au manque d'eau cités de manière plus régulière. Dans le cas du forum de val de Loire, une collecte de mots-clés par smartphone a été réalisée pour répondre à la question « le changement climatique pour vous c'est... » (Touzard et al., 2020). Les aléas climatiques et événements extrêmes (gel, phénomènes extrêmes, aléas climatiques, orage, stress) sont ressortis comme préoccupation importante $(15 \%$ des 153 mots-clés transmis), tout comme les effets liés à l'augmentation graduelle des températures sur la précocité de la vigne, le degré alcoolique et l'acidité des raisins (16\% des mots-clés). Enfin, le risque sécheresse est ressorti comme une préoccupation non négligeable $(8 \%)$. Ce sondage fait également ressortir l'importance des solutions agronomiques pour l'adaptation (14\%).

Les conséquences de chaque scénario d'adaptation, évaluées par les participants, ont été enregistrées et analysées de manière identique pour les sept forums. Les résultats, issus de verbatims énoncés par groupe de 5 à 8 personnes, synthétisés dans le tableau 4, révèlent les principaux éléments perçus comme positifs ou négatifs dans l'ensemble des régions. Cette synthèse est le résultat d'une analyse qualitative de l'ensemble des verbatims, réalisée par les membres du groupe prospective, d'abord pour chaque région (regroupement thématique), puis pour l'ensemble des régions. Si un élément ressort dans la synthèse globale, c'est qu'il a été cité au moins une fois dans la majorité des régions (au moins 6 sur 7). Les éléments spécifiques à certaines régions n'ont été cités que dans ces régions et relèvent aussi d'une vision globale de l'ensemble des verbatims. Certains verbatims sont cités entre parenthèses et en italique à titre d'exemple. 
Tableau 4. Synthèse des conséquences évaluées et caractérisées comme positives ou négatives par les participants aux sept forums régionaux des quatre scénarios d'adaptation de la filière vitivinicole. Summary of the consequences evaluated and qualified as positive or negative by the participants to the seven regional forums, for the four scenarios of adaptation for the wine industry.

\begin{tabular}{|c|c|c|}
\hline $\begin{array}{c}\text { Scénario } \\
\text { d'adaptation }\end{array}$ & $\begin{array}{l}\text { Conséquences estimées } \\
\text { comme positives }\end{array}$ & $\begin{array}{l}\text { Conséquences estimées } \\
\text { comme négatives }\end{array}$ \\
\hline Conservateur & $\begin{array}{l}\text { - Valorisation de la diversité } \\
\text { - Environnement } \\
\text { - La filière française comme référence }\end{array}$ & $\begin{array}{l}\text { - Diminution du rendement et qualité } \\
\text { variable } \\
\text { - Pas d'amélioration technique } \\
\text { - Prix élevé des vins } \\
\text { - Perte de compétitivité }\end{array}$ \\
\hline Innovant & $\begin{array}{l}\text { - Nombreuses innovations : matériel végétal, } \\
\text { œnologie, marketing, emballages, services, } \\
\text { types de vins, etc... } \\
\text { - Combinaisons de modèles de viticulture } \\
\text { classique, biologique, précision } \\
\text { - Modification des cahiers des charges AOC }\end{array}$ & $\begin{array}{l}\text { - Besoin de financements } \\
\text { - Autres types d'investisseurs non } \\
\text { traditionnels } \\
\text { - Perte des références historiques } \\
\text { - Perte d'influence du système des AOC }\end{array}$ \\
\hline Nomade & $\begin{array}{l}\text { - Opportunités pour de nouvelles } \\
\text { combinaisons sol/climat/cépages/pratiques } \\
\text { - Opportunités pour de nouveaux marchés } \\
\text { - Nouveaux acteurs } \\
\text { - Elargissement des zones irrigables }\end{array}$ & $\begin{array}{l}\text { - Fragilité des vignobles traditionnels et des } \\
\text { activités associées (oenotourisme) } \\
\text { - Risques élevés pour la qualité } \\
\text { - Fin du système des AOC } \\
\text { - Système basé sur la marque } \\
\text { - Concentration d'acteurs privés : } \\
\text { marketing, R\&D, services, vignobles }\end{array}$ \\
\hline Libéral & $\begin{array}{l}\text { - Moins de charges administratives } \\
\text { - Innovations soutenant la qualité et les } \\
\text { rendements } \\
\text { - Nouveaux vins, marchés, consommateurs, } \\
\text { emplois, investissements } \\
\text { - R\&D privatisée }\end{array}$ & $\begin{array}{l}\text { - Exclusion des petits vignerons des } \\
\text { services privés } \\
\text { - Industrialisation et perte de diversité et de } \\
\text { typicité } \\
\text { - Fin du système AOP, moins de vignobles } \\
\text { de coteaux } \\
\text { - Modifications de la gouvernance avec } \\
\text { perte de l'influence des producteurs de } \\
\text { raisins } \\
\text { - Concentration des producteurs, du } \\
\text { négoce, des acteurs de la vinification }\end{array}$ \\
\hline
\end{tabular}

- Pour la majorité des régions, le scénario conservateur a été considéré comme ayant des conséquences négatives pour les rendements (baisse de production, variabilité des volumes) et la qualité des vins (degré alcool élevé, baisse de l'acidité), qui seraient toujours plus impactés par les aléas climatiques et la variabilité interannuelle dans le contexte du changement climatique. La compétitivité de la filière en serait globalement affectée (diminution du nombre d'actifs, accroissement des coûts, rétraction de la production). Pour autant, ce scénario est présenté comme favorable au maintien de la diversité des terroirs et des vins, et à la protection de l'environnement. Il serait finalement à même de protéger « le modèle français de production de vins» (retour des cépages ancestraux, moins d'intrants, développement de l'oenotourisme). 
- Le scénario innovant a été perçu comme favorisant l'intégration d'innovations dans tous les domaines techniques (irrigation, variétés résistantes, robotisation, agroforesterie) et économiques (évolution du cahier des charges, renforcement $R \& D$ ) avec des options qui autorisent la cohabitation entre plusieurs modèles de viticulture (conventionnelle, de précision, biologique...). Mais ce scénario d'adaptation est aussi considéré comme couteux (hausse des coûts de production, investissements en hausse), reposant sur de nombreux investissements et mettant en danger le cadre réglementaire des appellations d'origine (regroupement des organismes de gestion - Interprofessions, ODGs, INAO - sous un seul acteur).

- Le scénario nomade est associé à de nouvelles opportunités en matière technique (nouvelles associations sol-climat-matériel végétal-pratiques, nouveaux vignobles) et commerciale, et à l'extension des zones irrigables et de nouveaux « terroirs » (nouveaux vignobles, plaines irrigables, diversification de l'offre). Mais il pourrait fragiliser des vignobles traditionnels (déprise agricole) et le secteur de l'œnotourisme (le terroir disparait), conduire au développement du système des marques et à la concentration des entreprises (standardisation des profils des vins, tissu social diminué).

- Enfin, le scénario libéral est perçu comme favorisant la réduction des charges administratives (moins de déclarations administratives) et le développement de nouvelles opportunités privées (diversification des modèles d'entreprise). Mais il apparaît aussi comme menaçant pour le modèle de viticulture familiale français (disparition des exploitations familiales, le commerce décide) et le système des appellations, ainsi que pour la gouvernance actuelle de la filière (disparition des interprofessions, gouvernance de l'aval) du fait notamment d'une concentration des entreprises à tous les niveaux (disparition des petites exploitations).

Des spécificités régionales peuvent être déjà dégagées dans l'analyse de ces réactions collectives aux quatre scénarios d'adaptation. L'inquiétude et le caractère d'urgence face au changement climatique étaient ainsi plus marqués dans les vignobles méridionaux (les petits disparaissent, perte de technicité, 2000 ans d'histoire de la viticulture rayée de la carte, le terroir disparait), conduisant à une plus faible distinction entre les quatre scénarios et à une vision plus pessimiste quel que soit le futur envisagé. Le « désarroi » était même plus marqué dans les Côtes du Rhône fortement structurées par le cadre réglementaire des Appellations, alors que l'exploration de solutions nouvelles est ressortie comme une opportunité pour les vignobles du Languedoc où les modèles économiques et types de vins sont plus diversifiés.

En Champagne, les inquiétudes portaient sur les risques que les différents scénarios pourraient faire peser sur l'organisation actuelle de la filière très structurée par son interprofession et particulièrement performante au le plan technique et économique (affaiblissement de l'interprofession, le Comité Interprofessionnel des Vins de Champagne disparait, disparition des structures collectives).

En Bourgogne et Beaujolais, la tension sur le foncier est apparue comme plus préoccupante (prix du foncier augmente, le foncier n'est plus détenu par les exploitants, pression foncière).

Dans le Bordelais, des aspects positifs du changement climatique ont été identifiés pour tous les scénarios d'adaptation, prolongeant une perception générale de meilleurs millésimes sur la période récente (maîtrise technologique de la production, la région gagne, nouveaux territoires viticoles, pilotage par l'aval plus efficient). Les craintes sur l'évolution de qualité des vins sont pour autant mentionnées, avec un accent sur l'importance du négoce (perte de typicité, uniformisation des produits, pertes de repères des consommateurs).

En Alsace, l'attachement au modèle sociotechnique actuel s'est avéré prépondérant, avec la volonté de faire évoluer les pratiques dans le cadre actuel en combinant enjeu d'adaptation au changement climatique et enjeu environnemental (renforcement du bio, traitements plus ciblés, viticulture productiviste, donc risque environnemental élevé, gestion de l'environnement plus difficile). L'option nomade est peu considérée comme une opportunité pour le vignoble alsacien.

Le Val de Loire a finalement montré une vision plus optimiste de l'avenir quels que soient les 
scénarios, le vignoble étant perçu comme climatiquement moins menacé que les vignobles méridionaux ou que d'autres vignobles septentrionaux plus spécialisés (changement climatique $=$ atout, la difficulté engendre la créativité, viticulture plus réfléchie, opportunité de nouveaux marchés, Val de Loire attractif).

\subsubsection{Les attitudes stratégiques des participants au regard des scénarios d'adaptation}

Après l'analyse des conséquences des 4 scénarios d'adaptation, les participants ont été appelés à s'exprimer sur l'attitude stratégique qu'ils souhaiteraient adopter vis-à-vis de chacun d'entre eux. Les résultats sont présentés dans le tableau 5.

Tableau 5. Attitudes stratégiques pour chacun des scénarios d'adaptation (\% des participants à chaque forum). Les votes pour chaque scénario sont indépendants. Les couleurs varient du rouge foncé au vert foncé en fonction du pourcentage (du plus faible au plus élevé ( $<5$, de 5 à 13 , de 14 à 25 , de 26 à 50 , de 50 à $75,>75)$. Strategic attitudes for each adaptive scenario (\% of participants to each forum). Votes are independent for each scenario. Colours are depending on the \% (<5, de 5 à 13, de 14 à 25, de 26 à 50, de 50 à 75, >75) from dark red (low percentage) to dark green (high percentage).

\begin{tabular}{|c|c|c|c|c|c|}
\hline \multirow{3}{*}{ Attitudes stratégiques } & \multirow[b]{3}{*}{ Régions } & \multirow{2}{*}{\multicolumn{4}{|c|}{ Scénarios d'adaptation }} \\
\hline & & & & & \\
\hline & & conservateur & innovant & nomade & libéral \\
\hline \multirow{7}{*}{ Proactivité positive } & Alsace & 29,3 & 56,1 & 0 & 2,5 \\
\hline & Bordelais & 13,3 & 81,7 & 1,6 & 5 \\
\hline & Beaujolais-Bourgogne & 7,5 & 82,5 & 5 & 7,5 \\
\hline & Champagne & 29,4 & 66,7 & 2 & 3,9 \\
\hline & Côtes du Rhône & 16,9 & 63,6 & 2,6 & 1,3 \\
\hline & Languedoc & 23,9 & 81,7 & 4,3 & 5,6 \\
\hline & Val de Loire & 23 & 77 & 7 & 6 \\
\hline \multirow{7}{*}{ Proactivité négative } & Alsace & 14,6 & 0 & 61 & 60 \\
\hline & Bordelais & 23,3 & 1,6 & 26,7 & 43,3 \\
\hline & Beaujolais-Bourgogne & 47,5 & 0 & 35 & 50 \\
\hline & Champagne & 43,1 & 2 & 58,8 & 66,7 \\
\hline & Côtes du Rhône & 44,3 & 6,5 & 36,8 & 71,1 \\
\hline & Languedoc & 21,1 & 1,4 & 31,9 & 52,1 \\
\hline & Val de Loire & 21 & 5 & 39 & 66 \\
\hline \multirow{7}{*}{ Réactivité anticipée } & Alsace & 36,6 & 39 & 12,2 & 25 \\
\hline & Bordelais & 36,7 & 16,7 & 33,3 & 21,7 \\
\hline & Beaujolais-Bourgogne & 20 & 15 & 32,5 & 15 \\
\hline & Champagne & 21,6 & 29,4 & 11,8 & 9,8 \\
\hline & Côtes du Rhône & 31,2 & 29,9 & 34,2 & 13,2 \\
\hline & Languedoc & 18,3 & 14,1 & 39,1 & 21,1 \\
\hline & Val de Loire & 42 & 17 & 33 & 12 \\
\hline \multirow{7}{*}{ Veille } & Alsace & 17,1 & 4,9 & 26,8 & 12,5 \\
\hline & Bordelais & 21,7 & 0 & 38,4 & 28,4 \\
\hline & Beaujolais-Bourgogne & 17,5 & 2,5 & 27,5 & 25 \\
\hline & Champagne & 5,9 & 2 & 27,5 & 17,6 \\
\hline & Côtes du Rhône & 7,3 & 0 & 26,3 & 14,5 \\
\hline & Languedoc & 28,2 & 2,8 & 23,2 & 21,1 \\
\hline & Val de Loire & 14 & 0 & 21 & 12 \\
\hline
\end{tabular}


La proactivité positive vis-à-vis du scénario conservateur dépasse $20 \%$ en Alsace, Champagne, Languedoc et Val de Loire. Elle est inférieure dans toutes les autres régions, et la plus faible en Bourgogne-Beaujolais. La proactivité négative visà-vis de ce scénario est proche de $45 \%$ pour trois régions (Beaujolais-Bourgogne, Champagne, Côtes du Rhône), légèrement supérieure à $20 \%$ dans trois régions (Bordelais, Languedoc, Val de Loire) et inférieure à $15 \%$ en Alsace. Le pourcentage de réactivité anticipée est très élevé en Val de Loire (42\%), intermédiaire dans trois autres régions (Bordelais, Alsace, Côtes du Rhône) et plus faible en Beaujolais-Bourgogne, Languedoc et Champagne. L'attitude de veille est la plus marquée en Languedoc et la plus faible en Champagne et Côtes du Rhône.

Pour le scénario innovant, on relève un très fort pourcentage de proactivité positive pour l'ensemble des régions viticoles. Trois régions sont marquées par des valeurs supérieures à $80 \%$ (Bordelais, Beaujolais-Bourgogne, Languedoc), trois autres affichent des taux compris entre 56 et $67 \%$ (Alsace, Champagne, Côtes du Rhône) et une dernière présente une valeur intermédiaire à $77 \%$ (Val de Loire). Les pourcentages de proactivité négative sont tous inférieurs à $7 \%$, mais l'attitude de réactivité anticipée vis-à-vis de ce scénario révèle deux groupes : l'un avec des pourcentages proches ou supérieurs à $30 \%$ (Alsace, Champagne, Côtes du Rhône) et le second avec des pourcentages voisins de $15 \%$ (Bordelais, Bourgogne-Beaujolais, Languedoc, Val de Loire). L'option de veille est faible pour toutes les régions.

Vis-à-vis du scénario nomade, la proactivité positive est très faible pour tous les vignobles. La proactivité négative est la plus forte en Alsace et en Champagne, autour de 30-40 \% dans quatre autres régions, et inférieure à $30 \%$ pour le Bordelais. La réactivité anticipée structure les régions de manière inversée. Globalement, le pourcentage de veille est voisin de $25 \%$ pour toutes les régions et un peu plus élevé dans le Bordelais.

Le pourcentage ne dépasse jamais $10 \%$ pour la proactivité positive vis-à-vis du scénario libéral. Pour la proactivité négative, il est au contraire élevé, mais «seulement» de $43 \%$ dans le Bordelais et autour de $50 \%$ en Beaujolais-Bourgogne et Languedoc, alors qu'il est supérieur à $60 \%$ en
Alsace, Champagne et Val de Loire et le plus élevé en Côtes du Rhône (71 \%). La réactivité anticipée est un peu plus élevée en Alsace, Bordelais et Languedoc (> à $20 \%$ ) par rapport aux autres régions. Enfin, une attitude de veille est plus marquée dans le Bordelais, en BourgogneBeaujolais et Languedoc (> $20 \%$ ) par rapport aux autres régions.

\subsubsection{Les propositions de leviers d'action}

Sur l'ensemble des sept forums, plus de 2600 propositions d'action ont été faites par les participants. Afin de donner une idée synthétique, ces propositions ont été catégorisées et les pourcentages de participants ayant fait ce type de propositions dans chacune des régions sont reportées dans le tableau 6. Des exemples d'action sont citées entre parenthèses et en italique.

S'il n'y a pas de différence notable entre régions pour les actions relatives à la $R \& D$, mises en avant partout, certaines spécificités régionales ressortent de notre analyse. L'Alsace se distingue par l'importance des actions associées aux catégories relatives à l'environnement (plus d'aide et de conseil pour l'évolution de la culture biologique/biodynamique), au choix des cépages (faire expérimentation sur nouveaux cépages résistants) et à la communication vis-à-vis des consommateurs. Le Bordelais est marquée par un intérêt plus soutenu vis-à-vis des pratiques œnologiques, la prise en compte des consommateurs (mettre en garde le consommateur/électeur), le marketing (anticiper les attentes des marchés et travailler l'image de nos produits) et également une attention élevée pour les questions d'encépagement (favoriser la diversité de l'encépagement). La Bourgogne-Beaujolais est proche du positionnement du Bordelais, mais s'en distingue par un intérêt un peu plus marqué pour les questions environnementales (avancer plus rapidement sur les questions environnementales) et surtout plus faible pour l'encépagement et les questions de réglementation. La Champagne est globalement caractérisée par de plus faibles pourcentages pour la plupart des catégories présentées et met en avant surtout la R\&D ainsi que le marketing et la communication. Les Côtes du Rhône et le Languedoc sont proches en mettant en avant l'irrigation (maîtrise des ressources en eau et de 
leur utilisation) et l'encépagement, et un intérêt accru pour les aspects environnementaux en Languedoc (accompagner les projets d'innovation qui respectent des « valeurs » environnementales/sociétales). Le Val de Loire est aussi marqué par des propositions d'actions en matière environnementale (positionner cette production dans une production très «naturelle » : biodynamie, agroforesterie, respect de la biodiversité, plantes bio-indicatrices, etc.).

Tableau 6. Catégories d'actions proposées pour alimenter l'attitude stratégique de proactivité positive pour les différents scénarios dans chacune des régions. Les valeurs correspondent au pourcentage de participants ayant fait des propositions dans ce domaine (d'après Ollat et Touzard, 2020). Les couleurs varient du rouge foncé (faible) au vert foncé (fort) en fonction du pourcentage. Categories of actions proposed by the participants to feed the proactive strategic attitudes identified for each scenario of adaptation, in each region. Numbers are the percentage of participants who made propositions belonging to these categories (from Ollat and Touzard, 2020). Colors are depending on the percentage from dark red (low percentage) to dark green (high percentage).

\begin{tabular}{|c|c|c|c|c|c|c|c|}
\hline Catégories d'action & Alsace & Bordelais & $\begin{array}{c}\text { Bourgogne- } \\
\text { Beaujolais }\end{array}$ & Champagne & $\begin{array}{c}\text { Côtes du } \\
\text { Rhône }\end{array}$ & Languedoc & Val de Loire \\
\hline $\begin{array}{c}\text { Recherche et } \\
\text { développement }\end{array}$ & 85 & 88 & 87 & 80 & 79 & 80 & 85 \\
\hline Réglementation & 54 & 54 & 41 & 36 & 46 & 52 & 51 \\
\hline $\begin{array}{c}\text { Communication et } \\
\text { marketing }\end{array}$ & 73 & 70 & 69 & 53 & 58 & 55 & 62 \\
\hline Terroir & 68 & 63 & 59 & 47 & 58 & 69 & 62 \\
\hline Environnement & 41 & 19 & 26 & 16 & 20 & 38 & 39 \\
\hline Encépagement & 46 & 40 & 23 & 22 & 46 & 37 & 34 \\
\hline Irrigation & 12 & 14 & 5 & 2 & 42 & 31 & 18 \\
\hline CEnologie & 19 & 25 & 23 & 11 & 14 & 23 & 24 \\
\hline
\end{tabular}

\section{Discussion}

L'objectif de cet article est de montrer comment une étude climatique conduite sur des périodes historique et future peut être associée à une démarche de prospective participative pour explorer des stratégies d'adaptation au changement climatique à des échelles régionales.

Pour cela, les perceptions du changement climatique par les acteurs de la filière vigne et vin et leurs réactions face à quatre scénarios d'adaptation ont été recueillies lors de journées de travail organisées dans sept régions viticoles françaises. L'étude climatique a permis de contextualiser et de confronter ces perceptions au regard des principales évolutions climatiques régionalisées. Elle apporte aussi une contribution aux travaux de construction de stratégies d'adaptation à l'échelle de filières régionales.

\subsection{Une régionalisation des évolutions climatiques pour mieux saisir les impacts sur les vignobles français}

Entre la fin du $\mathrm{XX}^{\mathrm{e}}$ et le début du $\mathrm{XXI}^{\mathrm{e}}$ siècle, les principales évolutions climatiques, estimées à l'aide d'indicateurs agroclimatiques fondés sur la température et les précipitations pendant la période végétative de la vigne se sont traduites dans les régions viticoles françaises essentiellement par une élévation des températures, conduisant à une hausse significative de l'indice héliothermique de Huglin de 130 à $230^{\circ} \mathrm{C}$.J environ. Les régions viticoles de climat "très frais", au sens proposé par Tonietto et Carbonneau en 2004, ont disparu pour embrasser un climat viticole "frais" à "tempéré chaud". Le nombre de journées dites "de forte chaleur" a été marqué par une faible augmentation (de 0,5 à 1,8 jours supplémentaires), mais restent assez peu fréquentes en ce début du $\mathrm{XXI}^{\mathrm{e}}$ siècle. 
Soulignons que les valeurs absolues (et donc les classes correspondantes) de l'indice de Huglin calculées sur la période 1980-1999 avec les données SAFRAN sont inférieures de l'ordre de 100 à $300^{\circ} \mathrm{C}$.J à celles calculées pour la fin du $20^{\mathrm{e}}$ siècle à partir de données de stations climatiques en régions viticoles (Colmar, Bordeaux, ...) et rapportées dans la littérature (Huglin et Schneider, 1998 ; Tonietto, 1999). Cette différence a pour origine un biais de la base de données SAFRAN concernant les températures maximales sur l'ensemble de la France (Vidal et al., 2010). En effet, en été, saison durant laquelle il apparaît plus marqué, ces températures sont sous-estimées dans les données SAFRAN, respectivement de $1^{\prime}$ ordre de $-1,5$ à $-1^{\circ} \mathrm{C}$ en Bourgogne et en Champagne viticoles (Zito, 2021). Les températures maximales contribuant fortement au calcul de l'indice de Huglin, il n'est donc pas étonnant de constater des valeurs plus basses que celles calculées à partir de données issues de stations climatiques.

L'augmentation du nombre des journées chaudes est surtout marquée dans les vignobles du sud-est de la France (+1,8 jours). Cet indicateur est probablement lui aussi sous-évalué en raison du biais sur les températures maximales de la base de données SAFRAN mentionné ci-dessus. Concernant les précipitations, aucune tendance nette et significative n'est observée entre les deux périodes récentes, à l'exception d'un infléchissement du nombre de jours avec précipitations d'avril à juillet dans le Beaujolais.

Au cours des prochaines décennies, la poursuite du réchauffement devrait conduire à affecter substantiellement la vitiviniculture en France. Pour le scénario climatique RCP8.5 dans un futur proche (2046-2065), les simulations débiaisées et désagrégées à une résolution de 8 x $8 \mathrm{~km}$, indiquent de façon unanime pour l'ensemble des 22 GCM considérés, une nette augmentation de l'indice de Huglin (+662 à $771^{\circ} \mathrm{C}$.J par rapport à la période 1980-1999) et du nombre de jours de fortes chaleur (+5,6 à 22,6 jours) dans la totalité des régions viticoles étudiées. Cette hausse des températures est surtout remarquable dans le sud-est de la France et en Beaujolais avec des modifications significatives des indicateurs climatiques associés, projetant la vitiviniculture française vers des conditions climatiques inédites qui devraient affecter nettement la croissance de la vigne et la maturation $\mathrm{du}$ raisin avec une accentuation des stress thermiques.

Concernant les précipitations, l'évolution attendue à cet horizon temporel reste incertaine, en raison de l'hétérogénéité des climats simulés par les 22 modèles de circulation générale dans le cadre de 1'exercice CMIP5. Bien que les cumuls de précipitation d'avril à septembre et d'avril à juillet présentent une tendance à la baisse dans toutes les régions, sauf en Alsace, ces variations ne sont pas significatives dans les régions étudiées pour l'horizon temporel considéré. Les réductions les plus marquées concerneraient les vignobles méridionaux (en dessous du $45^{\mathrm{e}}$ parallèle $\mathrm{N}$ ). Cette évolution faible, souvent non significative, des précipitations sur la période récente et d'ici le milieu du $\mathrm{XXI}^{\mathrm{e}}$ siècle est confirmée par plusieurs auteurs, même dans les régions méridionales (Schultz, 2017 ; Soubeyroux et al., 2021). Il est cependant admis qu'une augmentation de la température conduit à une augmentation de la demande évaporative, notamment pendant l'été qui peut provoquer des situations de sécheresse du sol plus marquée. Bien que l'évapotranspiration potentielle soit également tributaire d'autres éléments du climat, comme le vent dont la diminution a entrainé une baisse (contre-intuitive, dans un climat plus chaud) de la demande évaporative dans plusieurs régions du monde (McVicar et al., 2012), on observe jusqu'à ce jour une élévation en Europe de cette demande (Schultz, 2017). Pieri (2010) rapporte une baisse potentielle de la restitution d'eau au milieu sous vigne tout au cours du $\mathrm{XXI}^{\mathrm{e}}$ siècle dans la majorité des régions viticoles, confirmée sur le passé récent en zone méditerranéenne (Saurin, comm. pers.). Lebon et Garcia de Cortazar-Atauri (2014) projettent quant à eux une augmentation de la durée de la période de déficit hydrique d'environ un mois pour le vignoble languedocien.

\subsection{La confirmation d'une géographie de la perception du changement climatique}

Les évolutions climatiques récentes se sont essentiellement manifestées par une élévation des températures conduisant à une plus grande précocité du cycle de la vigne, de la récolte et de la maturation 
du raisin (Lereboullet et al., 2013 ; van Leeuwen et Destrac-Irvine, 2017 ; Labbé et al., 2019). Ces modifications sont bien perçues par les acteurs professionnels, comme le montre l'analyse des verbatims des forums participatifs relativement aux risques climatiques et à leurs conséquences sur la vigne. Nos résultats confirment des observations faites depuis le début des années 2000 (Battaglini et $a l ., 2009)$. Une enquête conduite ensuite en 2009 révèle que les exploitants vitivinicoles et experts de la filière interrogés rapportaient comme principales conséquences du changement climatique en France une avancée de la date de récolte et une modification de la maturation du raisin (élévation de la teneur en sucres et baisse de l'acidité) en lien avec l'augmentation des températures (Rochard et $a l ., 2010)$. Dans cette même enquête, l'inquiétude vis-à-vis de cette évolution, mais également des risques de sècheresse, apparaissait davantage marquée dans les régions du sud de la France (Languedoc-Roussillon et Provence-Alpes-Côte d'Azur). Cette inquiétude a été confirmée pour le Roussillon où les années chaudes et sèches sont toujours perçues comme négatives pour les producteurs avec des rendements plus bas et des blocages de maturation (Lereboullet et al., 2013). Plusieurs enquêtes conduites auprès de viticulteurs du Languedoc entre 2014 et 2016, c'est-à-dire juste avant l'organisation du forum dans cette région, soulignent également la préoccupation majeure autour de la question de l'eau (Graveline et Grémont, 2021).

Cette géographie de la perception du changement climatique n'est pas totalement cohérente avec l'intensité du réchauffement entre la fin du $\mathrm{XX}^{\mathrm{e}}$ et début du $\mathrm{XXI}^{\mathrm{e}}$ siècle rapportée ici. En effet, l'augmentation récente des températures est plus marquée pour les vignobles septentrionaux. Pour ces régions septentrionales, comme en Alsace, le réchauffement climatique contemporain a pu être considéré comme favorable car il a permis d'atteindre des teneurs en sucres plus élevées et ainsi plus proches des teneurs souhaitées pour la vinification (Duchêne et Schneider, 2005). La perception des risques liés à la sécheresse parait plus aigüe que dans la réalité des faits, notamment dans les régions autres que méditerranéennes. La crainte d'une pression parasitaire accrue pour la vigne davantage mise en avant dans les vignobles septentrionaux n'est également pas confirmée dans les faits ou dans les projections pour lesquelles la variabilité est très forte, en lien avec celle des précipitations (Zito, 2021).

\subsection{Convergences et spécificités régionales pour l'évaluation des conséquences des scénarios d'adaptation}

Au-delà d'une contribution à l'analyse des perceptions du changement climatique, les sept forums participatifs ont permis d'explorer les stratégies d'adaptation déjà engagées ou possibles face à une gamme de situations climatiques variées, que ce soit actuellement ou pour les 30 prochaines années.

Ces forums se sont déroulés sur deux années et demie, ce qui peut être considéré comme une période longue entrainant un biais quant à la perception des acteurs. Mais les résultats obtenus dans le Bordelais (24/11/2016) et dans le Val de Loire (7/03/2019) ne révèlent pas d'écarts marqués de perception et d'évaluation des conséquences entre ces deux dates, ce qui limite les réserves à ce sujet. Les différences de représentativité des catégories socio-professionnelles entre les forums sont également à noter, avec certains forums marqués par une plus grande proportion de producteurs (Alsace, Champagne, Côtes du Rhône, Languedoc) et d'autres par une part plus importante de représentants de la catégorie "Recherche \& Développement » (Bordelais, Bourgogne-Beaujolais). Cela a pu bien sûr affecter globalement les résultats obtenus d'un forum à l'autre, en complément des autres variables non contrôlées. Cependant, pour ce type d'exercice, la présence suffisante d'acteurs dans les mêmes catégories (production, R\&Dconseil, gestion-administration) garantit une solidité et cohérence des résultats, y compris pour des comparaisons interrégionales. La faible représentation du négoce et de fournisseurs de la filière est générale et donne aux résultats une orientation centrée sur la production des vins dans chaque région. Le biais par rapport à la connaissance de l'enjeu climatique, en lien avec la participation volontaire à de tels forums, a été mentionné par ailleurs.

Les conséquences envisagées pour chacun des scénarios d'adaptation sont globalement les mêmes 
dans les sept régions viticoles, ainsi que les leviers à activer. Il faut d'abord constater une convergence entre les visions de l'avenir « dans le contexte du changement climatique en $2050 »$, avec dans toutes les régions un positionnement stratégique largement favorable à un scénario qui intégrerait de plus en plus d'innovations afin de conserver le modèle français de production et de valorisation du vin. Cette option, résumée par la formule « innover pour rester », exprime l'importance des investissements réalisés par les viticulteurs dans des ressources productives ancrées localement (pérennité des plantations, paysages, savoir et histoire, réputation liée à l'origine géographique...), qui donnent une valeur patrimoniale élevée aux vignobles, et qu'il convient de préserver... en innovant (Belletti et al., 2017 ; Aigrain et al., 2019). La quasi absence de proactivité positive pour les scenarios «nomade » et « libéral » est aussi commune à toutes les régions, avec un positionnement de veille partagé seulement vis-à-vis du scénario nomade, traduisant la même interrogation sur une stratégie déjà observée ponctuellement (Bonnardot et Quénol, 2020) et donc « à surveiller ». Le positionnement proactif négatif vis-à-vis du scenario libéral est général, certes un peu moins marqué pour le Bordelais, reflétant un attachement au maintien d'une régulation pour le secteur, confirmé par d'autre travaux (Hannin et al., 2021). Les convergences sont aussi repérées dans les actions proposées, avec des investissements plébiscités pour la $R \& D$ et l'expérimentation, conformes avec l'option innovante partagée par les régions, quelles que soient leurs évolutions climatiques. L'importance accordée aux actions dans les domaines du marketing, de la communication ou de la réglementation exprime aussi la perception que l'adaptation se joue au plan économique, notamment dans la capacité à valoriser des vins dont les qualités peuvent évoluer du fait du changement climatique (Ollat et al., 2016).

Certaines spécificités régionales sont cependant ressorties. Elles peuvent avoir des explications à la fois d'ordre climatique et socio-économique. Les vignobles se sentant les plus menacés sont bien les vignobles méridionaux, mais il est intéressant de noter que l'inquiétude concerne plus le paramètre hydrique que thermique, alors que les simulations climatiques révèlent bien que l'augmentation des températures (en moyenne annuelle ou lors de période de type «vague de chaleur») constitue la menace principale pour ces régions. L'importance accordée à l'irrigation et la centralité du thème de l'eau dans les propositions d'action de ces régions renforce cette spécificité, confirmant là encore les études menées en Languedoc (Graveline et Gramont, 2021). L'attachement à un scénario conservateur est plus fort pour les vignobles septentrionaux (Alsace, Champagne, Val de Loire) où enjeux patrimoniaux et perception d'avantages liés aux évolutions climatiques sont un peu plus marqués, mais la participation plus importante de producteurs aux différents forums pourrait aussi avoir contribué à ce résultat. Pour l'Alsace et la Champagne, l'engagement à agir pour contrer le développement du scénario nomade est toutefois nettement plus marqué qu'en Val de Loire où les acteurs ont exprimé un avantage possible à bénéficier de l'arrivée de viticulteurs « migrants climatiques » (Touzard et al., 2020). Les vignobles de la façade atlantique sont globalement apparus comme moins pessimistes, ce qui est cohérent avec le moindre réchauffement attendu dans ces régions, la diversité des vins produits et leurs capacités adaptatives d'ordre économique, commerciale ou liée à leur organisation professionnelle (Boyer et Touzard, 2016). Les propositions d'action amènent surtout à distinguer la Champagne. Les domaines liés à l'œnologie, aux consommateurs, aux terroirs ou même à l'environnement y sont moins présents, exprimant le fait que ces leviers sont de fait déjà activés ou pensent être maitrisés par les acteurs d'un système de production et de commercialisation (vin effervescent, stratégies de marques) qui s'est construit historiquement précisément pour faire face à des aléas climatiques plus nombreux. L'importance et la performance de la R\&D champenoise, impulsée par son interprofession, ressort aussi dans d'autres études comme un facteur majeur pour l'adaptation de ce vignoble (Boyer et Touzard, 2021) pouvant expliquer un climat de confiance des acteurs de cette filière. Les positionnements et options des régions viticoles face au changement climatique expriment ainsi largement des conditions socioéconomiques (on parle de « système régionaux d'innovation ॥; Boyer, 2016) qui ne sont pas directement liées aux évolutions climatiques récentes ou envisagées par les simulations. 


\section{Conclusion}

L'objectif de ces forums régionaux était avant tout d'explorer la diversité des situations, des engagements d'actions et des stratégies d'adaptation possibles des vignobles français vis-àvis du changement climatique. La réalisation d'une étude climatique, sur des données historiques et simulées pour l'horizon 2050, a permis de contextualiser, mais aussi de questionner les résultats de ces forums, ouvrant la perspective d'une analyse plus poussée pour expliquer la diversité des positionnements des acteurs. Les réactions et propositions dans chaque région reflètent pour partie une cohérence entre étude climatique et perceptions de ces évolutions par les acteurs (effets des influences atlantiques, méditerranéennes, septentrionales et continentales). Mais l'article montre aussi que ces réactions peuvent être marquées i) par des perceptions qui ne correspondent pas partout à toutes les dimensions des évolutions climatiques étudiées (par exemple, la question de l'eau présente partout et «excessivement » prégnante dans les vignobles méridionaux) et $i$ ) par des conditions de production et d'organisation de la filière qui jouent sur les visions, capacités et orientations de l'adaptation, à l'image de la région Champagne très engagée autour de son interprofession. Ces hypothèses mériteraient d'être vérifiées, mais elles rejoignent d'autres études récentes. $\mathrm{La}$ richesse des informations recueillies a cependant permis de se forger une représentation très utile de la diversité des perceptions régionales de l'enjeu climatique au sein de la filière viticole. Le matériau recueilli a en effet nourri l'élaboration d'une stratégie nationale de la filière pour faire face aux enjeux climatiques (INAO et al., 2021). Les positions des acteurs des régions viticoles et leurs propositions ont été catégorisées en domaines d'action plus précis, pour construire un document stratégique présenté au Ministre de l'agriculture le 26 août 2021.

Remerciements : les auteurs remercient le Métaprogramme INRAE ACCAF qui a financé les travaux de prospective dans le cadre du projet Laccave, la KIC-Climat qui a contribué à l'organisation des forums participatifs, ainsi que tous les organismes professionnels et régionaux qui ont co-organisé et participé financièrement aux forums.

\section{Références}

Aigrain P., Brugière F., Duchêne E., Garcia de CortazarAtauri., Gautier J., Giraud-Héraud E., Hannin H., Ollat N., Touzard J-M., 2016a. Adaptation au changement climatique : intérêt d'une démarche prospective. BIO Web of Conferences, 7. DOI:10.1051/bioconf/20160703015

Aigrain P., Brugière F., Duchêne E., Garcia De Cortazar Atauri I., Gautier J., Giraud-Héraud E., Hannin,H., Ollat N., Touzard J.-M., 2016b. Travaux de prospective sur l'adaptation de la viticulture au changement climatique : quelles séries d'événements pourraient favoriser différentes stratégies d'adaptation ? BIO Web of Conferences, 7. DOI:10.1051/bioconf/20160703016

Aigrain P., Brugière F., Duchêne E., Garcia De Cortazar Atauri I., Gautier J., Ollat N., Giraud-Heraud E., Hannin H., Touzard J.-M., 2016c. Une prospective pour le secteur vignes et vins dans le contexte du changement climatique. Les synthèses de FranceAgriMer, 40, 21 p., https://hal.inrae.fr/hal01506534

Aigrain P., Bois B., Brugière F., Duchêne E., Garcia de Cortazar-Atauri I., Gautier J., Giraud-Héraud E., Hammond R., Hannin H., Ollat N., Touzard J-M., 2019. L'utilisation par la viticulture française d'un exercice de prospective pour l'élaboration d'une stratégie d'adaptation au changement climatique. BIO Web of Conferences, 12. DOI:10.1051/bioconf/20191203020

Battaglini A., Barbeau G., Bindi M., Badeck F.-W., 2009. European wingrowers'perception of climate change impact and options for adaptation. Reg. Environ. Change, 9, 61-73.

Belletti G., Marescotti A., Touzard J.-M., 2017. Geographical indications, public goods, and sustainable development: the roles of actors' strategies and public policies. World Development, 98 , 45-57, dx.doi.org/10.1016/j.worlddev.2015.05.004

Beltrando G., Briche E., 2010. Changement climatique et viticulture en Champagne : du constat actuel aux prévisions du modèle ARPEGE-Climat sur l'évolution des températures pour le $\mathrm{XXI}^{\mathrm{e}}$ siècle. EchoGéo, 14, 1-16.

Bois B., 2019. Assessment of climate change impacts on water availability for vitiviniculture worldwide using different potential evapotranspiration methods. 21st GiESCO International Meeting: 'A Multidisciplinary Vision towards Sustainable Viticulture', 44-48, Thessaloniki: Stefanos Koundouras.

Bois B., 2020. Viticulture and climate: from global to local. Proceedings of the 13th International Terroir Congress, 8, Adelaide, Australia: University of Adelaide.

Bois B., Pieri P., Van Leeuwen C., Wald L., Huard F., Gaudillere J.-P., Saur E., 2008. Using remotely sensed solar radiation data for reference evapotranspiration estimation at a daily time step. Agricultural and Forest Meteorology, 148, 619-630.

Bois B., Zito S., Calonnec A., 2017. Climate vs grapevine 
pests and diseases worldwide: the first results of a global survey. OENO One, 51, 133-139.

Bonnardot V., Quénol H., 2020. Viticulture en Bretagne : challenge ou opportunité. Changement Climatique et Territoires, 127-132. 〈hal-02934910〉

Boyer J., 2016. L'implication des acteurs de la recherche dans les processus d'adaptation au changement climatique : l'exemple des régions viticoles françaises. Innovations, 51, 147-171. doi.org/10.3917/inno.051.0147

Boyer J., Touzard J.-M., 2016. Recherche, innovation et compétitivité des vignobles français : analyse par les institutions. Systèmes Alimentaires / Food Systems, 1, 69-96, hal.inrae.fr/hal-02629861

Boyer J., Touzard J.-M., 2021. To what extent do an innovation system and cleaner technological regime affect the decision-making process of climate change adaptation? Evidence from wine producers in three wine clusters in France. Journal of Cleaner Production, 315, 1-13, dx.doi.org/10.1016/j.jclepro.2021.128218

Briche E., Beltrando G., Kergomard C., Quénol H., 2009. Utilisation des sorties du modèle numérique ARPEGE-Climat pour la simulation d'un indice bioclimatique jusqu'à la fin du $\mathrm{XXI}^{\mathrm{è}}$ siècle dans le vignoble de Champagne. Neuvièmes Rencontres de Théo Quant, Besançon, 4-6 mars, www.thema.univ-fcomte.fr.

Briche E., 2011. Changement climatique dans le vignoble de Champagne : Modélisation thermique à plusieurs échelles spatio-temporelles (1950-2100). Thèse de doctorat, Université Paris-Diderot - Paris VII, 308 p.

Chan S. C., Kendon E. J., Berthou S., Fosser G., Lewis E., Fowler H. J., 2020. Europe-wide precipitation projections at convection permitting scale with the Unified Model. Climate Dynamics, 55, 409-428.

Duchêne E., Schneider C., 2005. Grapevine and climatic changes: a glance at the situation in Alsace. Agronomy for Sustainable Development, 25, 93-99.

Fraga H., Garcia de Cortázar Atauri I., Malheiro A. C., Santos J. A., 2016. Modelling climate change impacts on viticultural yield, phenology and stress conditions in Europe. Global Change Biology, 22, 3774-3788.

GIEC Climate Change 2021: The Physical Science Basis. Contribution of Working Group I to the Sixth Assessment Report of the Intergovernmental Panel on Climate Change (V. Masson-Delmotte, P. Zhai, A. Pirani, S. L. Connors, C. Péan, S. Berger, N. Caud, Y. Chen, M. I. Goldfard, M. I. Gomis, M. Huang, E. Leitzell, E. Lonnoy, J. B. R. Matthews, T. K. Maycock, T. Waterfield, O. Yelekçi, R. Yu et B. Zhou, Dir.). Cambridge (UK): Cambridge University Press.

Graveline N., Grémont M., 2021. The role of perceptions, goals and characteristics of wine growers on irrigation adoption in the context of climate change. Agricultural Water Management, 250, 106837, doi.org/10.1016/j.agwat.2021.106837.

Gudmundsson L., Bremnes J. B., Haugen J. E., EngenSkaugen T., 2012. Technical Note: Downscaling RCM precipitation to the station scale using statistical transformations; a comparison of methods. Hydrology and
Earth System Sciences, 16(9), 3383-3390. doi.org/10.5194/hess-16-3383-2012

Hannin H., Touzard J.-M., Aigrain P., Bois B., Brugière F., Duchêne E., Garcia de Cortazar-Atauri I., Gautier J., GiraudHéraud E., Hammond R., Ollat N., 2021. Le vignoble français face au changement climatique : l'élaboration d'une stratégie d'adaptation à partir de scénarios de prospective. In: Cheriet F., Hannin H., Maurel C., Amadieu P. (eds), Management et marketing du vin : opportunités pour les entreprises et enjeux pour la filière, Agriculture, Science des aliments et nutrition, ISTE Editions, 55-76.

Huglin P., 1978. Nouveau mode d'évaluation des possibilités héliothermiques d'un milieu viticole. Comptes Rendus de l'Académie d'Agriculture de France, 64, 1117-1126.

Huglin P. et Schneider C., 1998. Biologie et écologie de la vigne. Lavoisier, $370 \mathrm{p}$.

Hulands S., Greer D. H., Harper J. D. I, 2013. The Interactive Effects of Temperature and Light Intensity on Vitis vinifera cv. 'Semillon' Grapevines. I. Berry Growth and Development. European Journal or Horticultural Science, 78, 246-257.

Hulands S., Greer D. H., Harper J. D. I., 2014. The Interactive Effects of Temperature and Light Intensity on Vitis vinifera cv. 'Semillon' Grapevines. II. Berry Ripening and Susceptibility to Sunburn at Harvest. European Journal of Horticultural Science, v79, 1-7.

INAO, FranceAgriMer, INRAE, IFV, 2021. Stratégie de la filière viticole face au changement climatique. www.inrae.fr/actualites/filiere-viticole-presente-au-ministresa-strategie-dadaptation-au-changement-climatique

Jones G. V., Morondo M., Bois B., HallA., Duff A., 2009. Analysis of the spatial climate structure in viticulture regions worldwide. Bulletin de l'OIV, 82, 507-517.

Labbé T., Pfister C., Brönnimann S., Rousseau D., Franke J., Bois B., 2019. The longest homogeneous series of grape harvest dates and its significance for the understanding of past and present climate. Climate of the Past, 15, 1485-1501.

Lebon E., Garcia de Cortazar Atauri I., 2014. Dans un contexte de changement climatique, quels sont les impacts de la sécheresse sur la vigne et sur le devenir des vignobles? L'exemple du Languedoc. In: Viticulture et stress hydrique Carrefours de l'Innovation Agronomique, Montpellier, 2014. INRA, 1-12

Le Moigne P., 2002. Description de l'analyse des champs de surface sur la France par le système SAFRAN. CNRMGMM2-MC2, 30 p.

Lereboullet A.-L., Bardsley D., Beltrando G., 2013. Assessing vulnerability and framing adaptive options of two Mediterranean wine growing regions facing climate change: Roussillon (France) and McLaren Vale (Australia). EchoGéo, 23, doi.org/10.4000/echogeo.13384

Lionello P., Scarascia L., 2018. The relation between climate change in the Mediterranean region and global warming. Regional Environmental Change, 18, 1481-1493.

Madelin M., Bois B., Chabin J.-P., 2010. Modification des conditions de maturation du raisin en Bourgogne viticole liée au réchauffement climatique. L'exemple des vignobles de la 
Côte et des Hautes-Côtes de Beaune. EchoGéo, 14. doi.org/10.4000/echogeo.12176

McVicar T. R., Roderick M. L., Donohue R. J., Li L. T., Van Niel T. G., Thomas A., Grieser J., Jhajharia D., Himri Y., Mahowald N. M., Mescherskaya A. V., Kruger A. C., Rehman S., Dinpashoh Y., 2012. Global review and synthesis of trends in observed terrestrial near-surface wind speeds: Implications for evaporation. Journal of Hydrology, 416-417, 182-205.

Météo France 2021. Changement climatique en France, synthèse du 2502 2021. meteofrance.com

Ollat N., Touzard J.-M., 2014. Long-term adaptation to climate change in viticulture and enology: the LACCAVE project. Journal International des Sciences de la Vigne et $d u$ Vin, Spécial Laccave, 1-7.

Ollat N., Touzard J.-M., Van Leeuwen C., 2016. Climate Change Impacts and Adaptations: New Challenges for the Wine Industry. Journal of Wine Economics, 11, 1-11.

Ollat N., Aigrain P., Bois B., Brugière F., Duchêne E., García De Cortázar-Atauri I., Gautier J., Giraud-Héraud E., Hammond R., Hannin H., Touzard J.-M., 2020. A quoi pourrait ressembler la filière vigne et vin française en 2050 ? Une mobilisation concertée pour répondre à cet enjeu. Eds Pérard J., Wolikow C., Fluctuations climatiques et vignobles, du Néolithique à l'actuel : impacts, résilience et perspectives, Chaire Unesco ; LIR3S, 2020, 978-2-918173-29-8 〈hal03310789>

Ollat N., Touzard J.-M., 2020. The wine industry confronted by Climate Change - LACCAVE PROJECT - Horizon 2050. $\langle 10.15454 /$ ar8r-s466〉 〈hal-02548387〉

Peña-Angulo D., Vicente-Serrano S. M., Domínguez-Castro F., Murphy C., Reig F., Tramblay Y., Trigo R. M., Luna M. Y., Turco. M., Noguera I., Aznárez-Balta M., García-Herrera R., Tomas-BurgueraM., Kenawy A. E., 2020. Long-term precipitation in Southwestern Europe reveals no clear trend attributable to anthropogenic forcing. Environ. Res. Lett., 15, 094070.

Pérez-Rodríguez P., Soto-Gómez D., De La Calle I., LópezPeriago J. E., Paradelo M., 2016. Rainfall-induced removal of copper-based spray residues from vines. Ecotoxicology and Environmental Safety, 132, 304-310.

Pieri P., Lebon E., 2010. Changement climatique et culture de la vigne: l'essentiel des impacts. In: Ademe (ed.), Changement climatique, agriculture et forêt en France : simulations d'impacts sur les principales espèces, Le Livre Vert du projet CLIMATOR (2007-2010), 213-223.

Remenyi T. A., Rollins D. A., Love P. T., Earl N. O., Bindoff N. L., Harris. R. M. B., 2019. Australia's Wine Future - A Climate Atlas. Hobart, Tasmania, Australia: University of Tasmania.

Rochard J., Zaba S., Chèvre C., 2010. Evolution du climat et viticulture. Analyse de la perception des professionnels et des experts. Poster présenté au $33^{\mathrm{e}}$ Congrès Mondial de la Vigne et du Vin, 20-27juin 2010, Tbilissi (Géorgie).

Salinari F., Giosuè S., Tubiello F.N., Rettori A., Rossi V., Spanna F., Rosenzweig C., Gullino M.L., 2007. Downy mildew (Plasmopara viticola) epidemics on grapevine under climate change. Global Change Biology, 12(7), 1299-1307.

Schultz H. R., 2017. Issues to be considered for strategic adaptation to climate evolution. Is atmospheric evaporative demand changing? OENO One, 51, 107-114.

Schwalm C. R., Glendon S., Duffy P. B., 2020. RCP8.5 tracks cumulative $\mathrm{CO}_{2}$ emissions. Proceedings of the National Academy of Sciences, 117, 19656-19657.

Sebillotte M., Aigrain P., Hannin H., Sebillotte C., 2003. Prospective : Vignes et Vins. Scénarios et défis pour la recherche et les acteurs. Bilan et Prospectives, Inra Editions.

Seguin B., Garcia de Cortazar-Atauri I., 2005. Climate warming: Consequences for viticulture and the notion of 'terroirs' in Europe. In: Williams L. E. (ed.), Proceedings of the Seventh International Symposium on Grapevine Physiology and Biotechnology. Acta Horticulturae, 689, 6169.

Sgubin G., Swingedouw D., Dayon G., García de CortázarAtauri I., Ollat N., Pagé C. , van Leeuwen, C., 2018. The risk of tardive frost damage in French vineyards in a changing climate. Agricultural and Forest Meteorology, 250-251, 226242.

Simonovici M., 2019. Enquête Pratiques phytosanitaires en viticulture en 2016. Agreste Les Dossiers, 2019-2, 50.

Soubeyroux J.-M., Bernus L., Corre L. et al., 2021. Les nouvelles projections climatiques de référence DRIAS 2020 pour la Métropole. Météo France, 98 p.

Taylor K. E., Stouffer R. J., Meehl G. A., 2012. An overview of CMIP5 and the experiment design. Bulletin of the American Meteorological Society, 93(4), 485-498.

Tonietto J., 1999. Les macroclimats viticoles mondiaux et l'influence du mésoclimat sur la typicité de la Syrah et du Muscat de Hambourg dans le sud de la France. Thèse de Doctorat, Ecole Nationale Supérieure Agronomique de Montpellier, $216 \mathrm{p}$.

Tonietto J., Carbonneau A., 2004. A multicriteria climatic classification system for grape-growing regions worldwide. Agricultural and Forest Meteorology, 124, 81-97.

Touzard J.-M., Ollat N., Aigrain P., Bois B., Brugière F., Duchêne E., Garcia de Cortazar-Atauri I., Gautier J., Hammond R., Hannin H., 2020. La filière Vigne et Vin face au changement climatique : enseignements d'un forum de prospective pour le Val de Loire. Norois, 2(255), 83-89. doi:10.4000/norois.9897

van Leeuwen C., Destrac-Irvine A., 2017. Modified grape composition under climate change conditions requires adaptations in the vineyard. OENO One, 51, 147-154.

Vicente-Serrano S. M., Domínguez-Castro F., Murphy C., Hannaford J., Reig F., Peña-Angulo D. et al., 2021. Long-term variability and trends in meteorological droughts in Western Europe (1851-2018). International Journal of Climatology, 41, E690-E717.

Vidal J.-P., Martin E., Franchistéguy L., Baillon. M., Soubeyroux J.-M., 2010. A 50-year high-resolution atmospheric reanalysis over France with the Safran system. International Journal of Climatology, 30, 1627-1644. 
Webb L., Watt A., Hill T., Whiting J., Wigg F., Dunn G., Needs S., Barlow S., 2009. Extreme heat: managing grapevine response based on vineyard observations from the 2009 heatwave across south-eastern Australia. University of Melbourne, Australia.

White M. A., Diffenbaugh N. S., Jones G. V., Pal J. S., Giorgi F., 2006. Extreme heat reduces and shifts United States premium wine production in the 21st century. Proceedings of the National Academy of Sciences, 103, 11217-11222.
Zito S., Caffarra A., Richard Y., Castel T., Bois B., 2018. Climate change and vine protection: the case of mildews management in Burgundy. E3S Web of Conferences, 50, 01006, 1-5.

Zito S., 2021. Evolution du risque phytosanitaire au vignoble dans le nord-est de la France en lien avec le changement climatique : observations et modélisation. Cas de l'oüdium de la vigne. Thèse de doctorat. Université de Bourgogne, Dijon, $224 \mathrm{p}$.

Citation de l'article : Ollat N. et al., 2021. La diversité des vignobles français face au changement climatique : simulations climatiques et prospective participative. Climatologie, 18, 3 . 
Figure supplémentaire 1. Dispersion statistique entre les valeurs d'indicateurs agroclimatiques calculées à partir des données historiques sur les périodes 1980-1999 et 2000-2019 pour chacune des régions viticoles. La dispersion interannuelle est schématisée au moyen d'un box-plot. En ordonnées : Huglin $\left[{ }^{\circ} \mathrm{C} . J\right]=$ indice héliothermique de Huglin ; TASMAXs35 = nombre de jours dont la température maximale dépasse $35^{\circ} \mathrm{C}$; GSPR $[\mathrm{mm}]=$ cumul de précipitations d'avril à septembre ; DSPR [mm] = cumul de précipitations d'avril à juillet ; DSPRs1 = nombre de jours d'avril à juillet dont le cumul de précipitations est supérieur ou égal à $1 \mathrm{~mm}$. Les astérisques rouges indiquent une évolution significative des indicateurs sur la période 2000-2019 en comparaison avec la période 1980-1999 pour plus de $50 \%$ des points de grilles sélectionnés au sein des régions viticoles. Statistical dispersion between agro-climatic indicators calculated from historical data over the periods 1980-1999 and 2000-2019 for each of the wine-growing regions. The inter-annual dispersion is shown in a box-plot. On the ordinates: Huglin] = Huglin heliothermal index; TASMAXs35 = number of days with maximum temperature above $35^{\circ} \mathrm{C}$; GSPR $[\mathrm{mm}]=$ cumulative precipitation from April to September; $D S P R[\mathrm{~mm}]=$ cumulative precipitation from April to July; DSPRs1 = number of days from April to July with cumulative precipitation greater than or equal to $1 \mathrm{~mm}$. The red asterisks indicate a significant change in the indicators over the period 2000-2019 compared to the period 1980-1999 for more than 50\% of the selected grid points within the wine-growing regions.
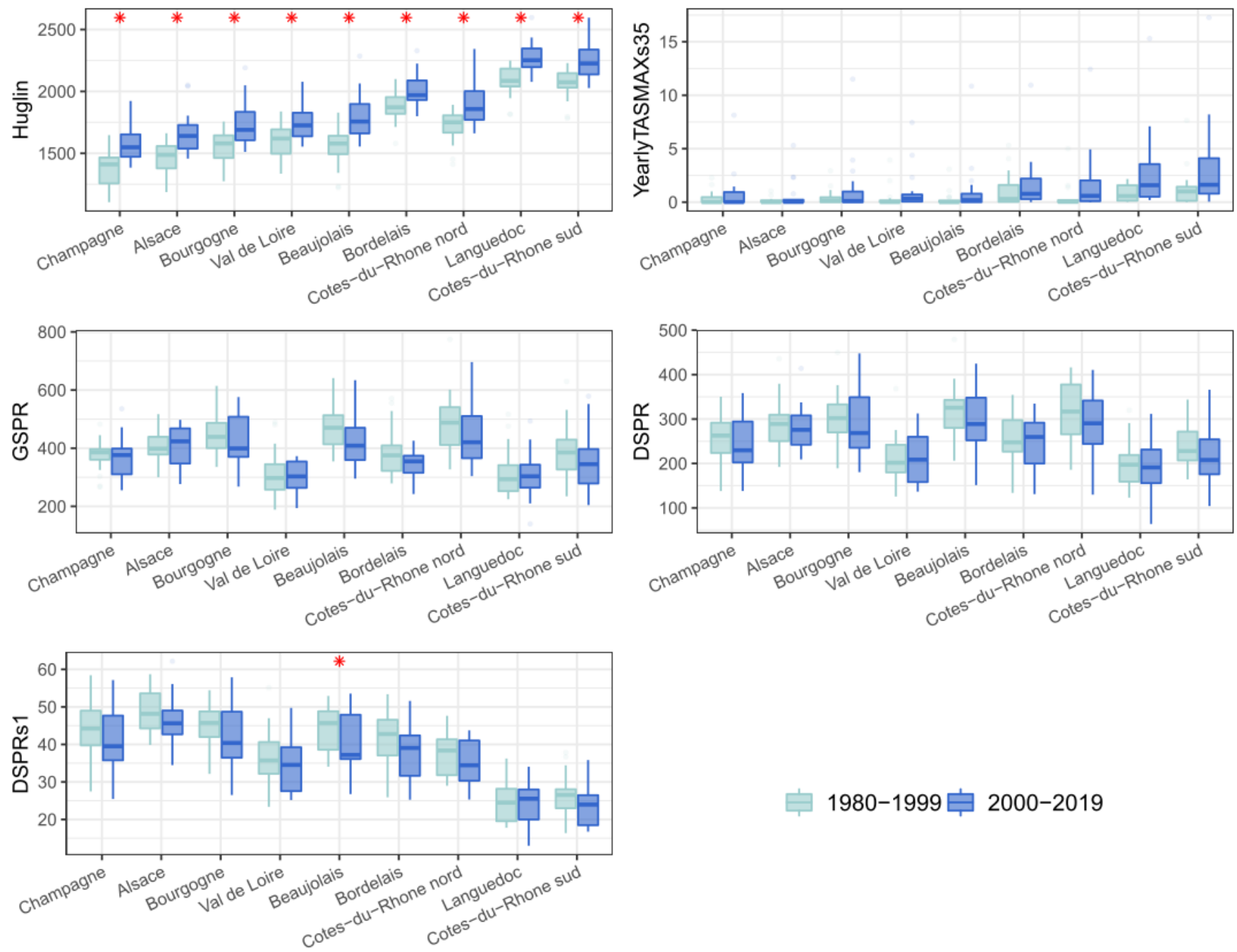